\title{
Slot-finger superconducting structure with rf focusing
}

\author{
Yu. Senichev and N. Vasyukhin \\ FZJ, Institute of Nuclear Physics, D-52425, Juelich, Germany
}

(Received 21 April 2005; published 12 July 2005)

\begin{abstract}
The linear accelerator based on a superconducting structure with a high accelerating gradient accordingly has a strong rf defocusing factor. Usually, a quadrupole or solenoid focusing system is used in such accelerators. Both of these systems complicate the linear accelerator. The quadrupoles are located outside the cavity and a transition between the cold and the warm systems is required. Therefore an additional drift space between cryostats is needed, which can cause parametric resonance in the longitudinal plane in the energy range of (3-20) $\mathrm{MeV}$. In the system with the solenoid the high magnetic field can affect the superconductivity. We consider the novel superconducting $\mathrm{H}$ resonator based on the TE211 mode with a slot and rf finger, providing the high-intensity beam focusing in the large range of low energy of (3-50) $\mathrm{MeV}$. Above $50 \mathrm{MeV}$ we suggest using the slot structure with the external quadrupoles.
\end{abstract}

DOI: 10.1103/PhysRevSTAB.8.070101

PACS numbers: 29.17.+w, 29.27.Bd, 41.75. $-\mathrm{i}$

\section{INTRODUCTION}

Normal-conducting structures with rf focusing have been extensively used during the last 30 years $[1,2]$. The advantages of accelerators without additional focusing elements are obvious. However, using the rf field in part for focusing, the normal-conducting resonator has lower shunt impedance, and due to the rf losses it cannot provide the high accelerating gradient in the accelerator with the high duty cycle. Therefore the superconducting cavity is the exclusive candidate for this class of machines. The first superconducting RFQ has been already been implemented [3], and the accelerator has demonstrated good prospects in the initial range of energy from $(0.05-0.1) \mathrm{MeV}$ up to (3-5) $\mathrm{MeV}$.

In this work, we discuss the novel slot-finger H-type superconducting structure, which promises to be good a candidate in the region $\sim(3-50) \mathrm{MeV}$. Above $50 \mathrm{MeV}$, in order to ensure a high accelerating gradient, it is reasonable to use the slot structure with the external quadrupoles. As distinct from the Teplyakov normal-conducting cavity based on the TE111 mode [2], we use the fundamental mode TE211. In the cavity with TE211 mode the electrodes are connected to the cavity at both ends, thus allowing the flowing electrodes to be used for the cooling liquid.

First of all, we consider the rf defocusing effect in the SC accelerator. Together with the higher accelerating rate in the superconducting linear accelerator the defocusing factor is much higher in comparison to the normal-conducting linear accelerator. Since the superconducting cavity is desirable to have a constant geometry, the quasisynchronous phase velocity is adjusted by the external rf phasing. The particles slide down or up relative to the rf wave as a function of the ratio between the particle and the wave velocity, and we can expect the rf defocusing factor to be modified. In this paper, we investigate how with external rf phasing the nonsynchronism affects the transverse motion through the rf defocusing factor.
As the first step to minimize the rf defocusing factor we introduce the quadrupole component in the accelerating gaps by two stems alternatively situated in the horizontal and vertical planes from gap to gap in the $\mathrm{H}$ resonator. Thus, instead of the drift tube, like in the spoke structure, we have vertical and horizontal slots between stems. The fundamental TE2 11 creates the $\pi$ mode in the longitudinal plane with the accelerating period $\beta \lambda / 2$ and the alternative rf quadrupole field with period $\beta \lambda$. Besides, the slot structure is simpler in comparison to the spoke resonator, since it does not require 3D brazing of the drift tubes.

However, the strength of the slot quadrupole field is not enough to provide beam stability for the appropriate synchronous phase value. Therefore we install two pairs of fingers in the first and last gaps of the resonator with two purely accelerating periods in the center of the cavity. Every second resonator is rotated around its axis to create a FOODDOOF focusing system, thus giving more stability in the transverse plane.

In the slot-finger accelerator we investigate the resonances between the longitudinal and transverse motions and the structural resonances due to the constant geometry of resonators belonging to one family.

Finally, we design the linear accelerator for (3-50) $\mathrm{MeV}$ with $40 \mathrm{~mA}$ current. We calculate the space charge tracking in the 3D model in order to investigate this effect.

\section{RADIO FREQUENCY DEFOCUSING IN STRUCTURE WITHOUT SYNCHRONISM}

\section{A. The single cavity}

First of all we consider the rf defocusing factor as the single integrated kick in one cavity with the axial symmetrical field and with the $n_{\text {cell }}$ absolutely identical accelerating cells, having the same sizes with periodicity $L_{\text {cell }}=\left(\beta_{\text {str }} \lambda / 2\right)$. The structure velocity $\beta_{\text {str }}$, some analogue of the phase velocity, is therefore constant in the cavity. The transverse motion equations are 


$$
\frac{d^{2} x}{d t^{2}}=\frac{e}{m_{0} \gamma^{3}} E_{x}, \quad \frac{d^{2} y}{d t^{2}}=\frac{e}{m_{0} \gamma^{3}} E_{y},
$$

where $E_{x, y}$ is the transverse component of the rf field. On the cavity axis the radial rf field can be represented as

$$
E_{x}(x)=E_{x}(0)+\frac{\partial E_{x}}{\partial x} \cdot x, \quad E_{y}(y)=E_{y}(0)+\frac{\partial E_{y}}{\partial y} \cdot y .
$$

In the case of the field with the axial symmetry we have $\left(\partial E_{x} / \partial x\right)=\left(\partial E_{y} / \partial y\right)$ and $E_{x}(0)=E_{y}(0)=0$.

Substituting the latter in the Maxwell equation $\left(\partial E_{x} / \partial x\right)+\left(\partial E_{y} / \partial y\right)+\left(\partial E_{z} / \partial z\right)=0$, we can easily get the ratio between the transverse and the longitudinal components

$$
\frac{\partial E_{x}}{\partial x}=\frac{\partial E_{y}}{\partial y}=-\frac{1}{2} \frac{\partial E_{z}}{\partial z} .
$$

For instance, in the $\mathrm{H}$ resonator with the angular frequency $\omega=(2 \pi c / \lambda)$ the accelerating field can be represented by the Fourier series

$$
E_{z}(z, t)=\sum_{n} E_{n} \sin \frac{2 \pi n}{\beta_{\mathrm{str}} \lambda} z \cdot \sin \left(\omega t+\varphi_{0}\right) .
$$

Then the motion Eqs. (1) can be rewritten in the longitudinal coordinates system $d z=c \beta d t$ :

$$
\begin{aligned}
& \frac{d^{2} x}{d z^{2}}=-\frac{e}{2 m_{0} c^{2} \gamma^{3} \beta^{2}} \cdot \sum_{n} k_{n} E_{n} \cos k_{n} z \cdot \sin \left(\omega t+\varphi_{0}\right) \cdot x, \\
& \frac{d^{2} y}{d z^{2}}=-\frac{e}{2 m_{0} c^{2} \gamma^{3} \beta^{2}} \cdot \sum_{n} k_{n} E_{n} \cos k_{n} z \cdot \sin \left(\omega t+\varphi_{0}\right) \cdot y,
\end{aligned}
$$

where $k_{n}=\left(2 \pi n / \beta_{\text {str }} \lambda\right)$ is the wave number of $n$th field harmonic.

Retaining in (4) the nearest harmonics to the quasisynchronous particle $E_{1} \cos k_{1} z$ with the wave number $k_{1}=$ $\left(2 \pi / \beta_{\text {str }} \lambda\right)$, we can use the traveling wave system. In this system, the phase of the arbitrary particle relative to the rf field is determined in accordance with the common definition:

$$
\begin{aligned}
\varphi(z, t) & =\omega t+\varphi_{0}-k_{1} z \\
& =\frac{2 \pi}{\lambda} \int_{0}^{z} \frac{d \xi}{\beta}+\varphi_{0}-\frac{2 \pi}{\lambda} \int_{0}^{z} \frac{d \xi}{\beta_{\mathrm{str}}},
\end{aligned}
$$

where $\varphi_{0}$ is the initial phase. In order to provide the quasisynchronism in the longitudinal plane the particle rf phase at the entrance and exit of the cavity has to be in the relation determined in [4]:

$$
\varphi_{L_{\text {cav }}}=\bar{\varphi}_{q s}+0.5 \cdot \Delta \varphi_{\text {slide }}, \quad \varphi_{0}=\bar{\varphi}_{q s}-0.5 \cdot \Delta \varphi_{\text {slide }},
$$

where $\Delta \varphi_{\text {slide }} \approx \pi n_{\text {cell }}\left[\left(\beta_{\text {str }} / \beta\right)-1\right]$ is the phase sliding factor due to the nonsynchronism, $\bar{\varphi}_{q s}$ is the average meaning of quasisynchronous phase.
Now we can calculate the trajectory-refracting angle of the quasisynchronous particle due to the rf defocusing effect over the whole cavity with the length of $L_{\text {cav }}=$ $\left(\beta_{\text {str }} \lambda / 2\right) \cdot n_{\text {cell }}$ :

$$
\Delta\left(\begin{array}{l}
\frac{d x}{d z} \\
\frac{d y}{d z}
\end{array}\right)=-\frac{e \pi k_{1} E_{1} \sin \bar{\varphi}_{q s}}{4 m_{0} c^{2} \gamma^{3} \beta^{2}} \cdot \frac{\sin \frac{\Delta \varphi_{\text {side }}}{2}}{\frac{\Delta \varphi_{\text {side }}}{2}} \cdot \frac{n_{\text {cell }} \beta_{\text {str }} \lambda}{2} \cdot\left(\begin{array}{l}
x \\
y
\end{array}\right) .
$$

In order to have stable longitudinal motion the average quasisynchronous phase has to be negative, that is to say the rf accelerating field always defocuses the particle. Let us call $T_{\text {slide }}=\left\{\left[\sin \left(\Delta \varphi_{\text {slide }} / 2\right)\right] /\left(\Delta \varphi_{\text {slide }} / 2\right)\right\}$ the time sliding factor. We can see that due to the nonsynchronism the rf defocusing factor decreases proportionally to the time sliding factor.

\section{B. Sequence of cavities with the constant geometry}

Equation (8) allows the rf defocusing integrating kick to be estimated when the particle is flying through the single cavity. In reality, the kick is modulated with the frequency of the cavities' repetition. Now we shall consider the case when the cavities are joined up in families, and all the $m$ cavities of one family have the same structure phase velocity $\beta_{\text {str }}=$ const for $i \in(1-m)$. Because of nonsynchronism the particle always slides in the cavity relative to the $\mathrm{rf}$ field and it is returned to the required rf phase at the entrance of next cavity by the rf phase shift. The rf phase shift law in each cavity $\varphi_{\mathrm{str}}(i)=\sum_{i} \Delta \varphi_{\mathrm{rf}}$ determines the quasisynchronous particle motion. Because of a proper choice of the rf phase shift $\Delta \varphi_{\mathrm{rf}}$ between cavities we can create a quasisynchronous motion and, in total, stable motion in the whole accelerator [4]. The quasisynchronous particle oscillating instantaneously in the single resonator around $\varphi=0$ (for "sin" wave) is forced by the intersection rf shift to oscillate on average around $\bar{\varphi}_{q s}$. Figure 1 shows how the quasisynchronous particle oscillates around the average value $\bar{\varphi}_{q s}$ in the linear accelerator with one family. For each section the initial and final phase deviations are not equal to each other.

Instead (6) the corrected phase of the quasisynchronous particle is $\varphi_{q s}(z)=(2 \pi / \lambda)\left[\int_{0}^{z}\left(d \xi / \beta_{q s}\right)-\int_{0}^{z}\left(d \xi / \beta_{\text {str }}\right)\right]+$ $\varphi_{\text {str }}(z)$ and the full equation system of quasisynchronous motion in the normalized coordinate system $\tau=z / \beta_{\text {str }} \lambda$ takes the form

$$
\begin{aligned}
\frac{d^{2} x}{d \tau^{2}} & =-\frac{e \lambda^{2} \beta_{\mathrm{str}}^{2}}{4 m_{0} c^{2} \gamma^{3} \beta_{q s}^{2}} \cdot k_{1} E_{1} \sin \varphi_{q s} \cdot x, \\
\frac{d^{2} y}{d \tau^{2}} & =-\frac{e \lambda^{2} \beta_{\mathrm{str}}^{2}}{4 m_{0} c^{2} \gamma^{3} \beta_{q s}^{2}} \cdot k_{1} E_{1} \sin \varphi_{q s} \cdot y, \\
\frac{d \varphi_{q s}}{d \tau} & =2 \pi\left(\frac{\beta_{\mathrm{str}}}{\beta_{q s}}-1\right)+\frac{d \varphi_{\mathrm{str}}}{d \tau}, \\
\frac{d \beta_{q s}}{d \tau} & =\frac{e E_{1} \lambda \beta_{\mathrm{str}}}{2 m_{0} c^{2} \gamma^{3} \beta_{q s}} \cos \varphi_{q s} .
\end{aligned}
$$




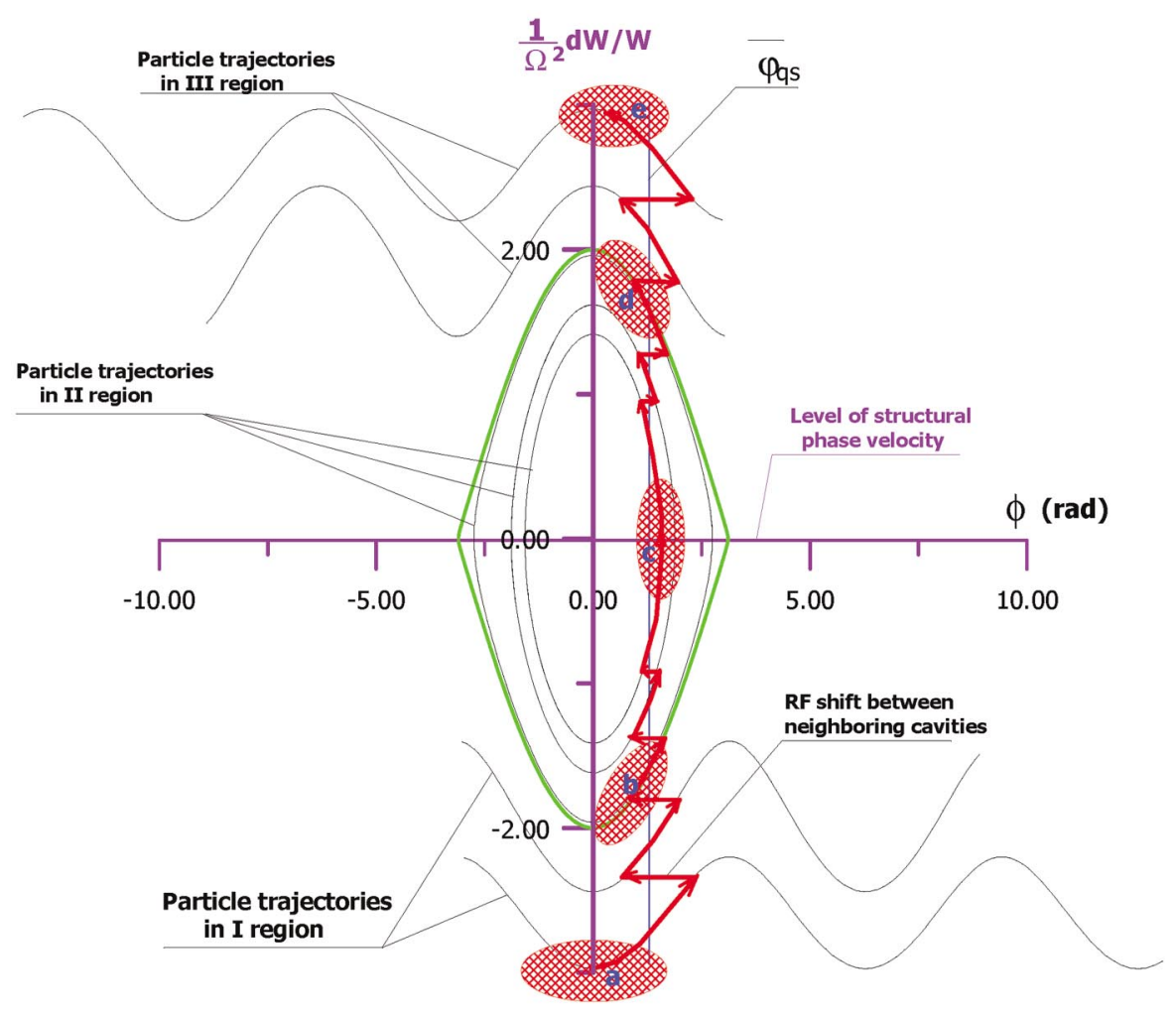

FIG. 1. (Color) Longitudinal motion in the stepped rf phase structure with one family.

The last two equations of the longitudinal motion are solved in [4] together with the determination of the optimum law for the rf phase shift. In particular, the instantaneous quasisynchronous phase is

$$
\begin{aligned}
\varphi_{q s}(\tau) & =\bar{\varphi}_{q s}+f(\tau) \\
\text { with } f(\tau) & =-2 \frac{d \bar{\varphi}_{\mathrm{str}}}{d \tau} \cdot \sum_{l=1}^{\infty} \frac{l \nu_{\mathrm{cav}} \cdot \sin \left(2 \pi l \nu_{\mathrm{cav}} \tau\right)}{\Omega^{2} \cos \bar{\varphi}_{q s}-l^{2} \nu_{\mathrm{cav}}^{2}}
\end{aligned}
$$

where $\nu_{\text {cav }}=1 / T_{\text {cav }}$ is the frequency of the rf phase shift and $\Omega$ is the longitudinal frequency in the sinus wave separatrix with $\varphi_{s}=0$. The period $T_{\text {cav }}=n_{\text {cell }} / 2$ measured in the number of $\beta_{\text {str }} \lambda$ has to coincide with the cavity length $L_{\text {cav }}=n_{\text {cell }} \beta_{\text {str }} \lambda / 2$.

For the practical case we can take $\nu_{\text {cav }} \gg \Omega \cos ^{1 / 2} \bar{\varphi}_{q s}$, and the first harmonic retains only $f(\tau)=\varphi_{a}(\tau)$. $\sin \left(2 \pi \nu_{\text {cav }} \tau\right)$ with the slowly time dependent amplitude $\varphi_{a}(\tau)=\left(2 / \nu_{\text {cav }}\right) \cdot\left(d \bar{\varphi}_{\text {str }} / d \tau\right)$. Thus, the quasisynchronous particle oscillates around the average value with the amplitude dependent on the nonsynchronism $\left(d \bar{\varphi}_{\text {str }} / d \tau\right)=$ $2 \pi\left\{1-\left[\beta_{\text {str }} / \beta(\tau)\right]\right\}$. For the higher relative velocity and the same number of cavities in one family the amplitude $\varphi_{a}(\tau)$ decreases.

Figure 2 shows the quasisynchronous phase oscillation versus the relative velocity in the case of 10 families with 6 cavities in each family. Substituting $\varphi_{q s}(\tau)=\bar{\varphi}_{q s}+f(\tau)$ in the transverse motion Eq. (9) and inserting the new coordinate $\xi=\left(\tau / T_{\text {cav }}\right)$ normalized to the cavity period- icity $T_{\text {cav }}=\left(n_{\text {cell }} / 2\right)$, we have, for instance, in the horizontal plane:

$$
\frac{d^{2} x}{d \xi^{2}}+\frac{A_{\mathrm{rf}} n_{\mathrm{cell}}^{2}}{4} \sin \left(\bar{\varphi}_{q s}+\varphi_{a} \sin 2 \pi \xi\right) \cdot x=0,
$$

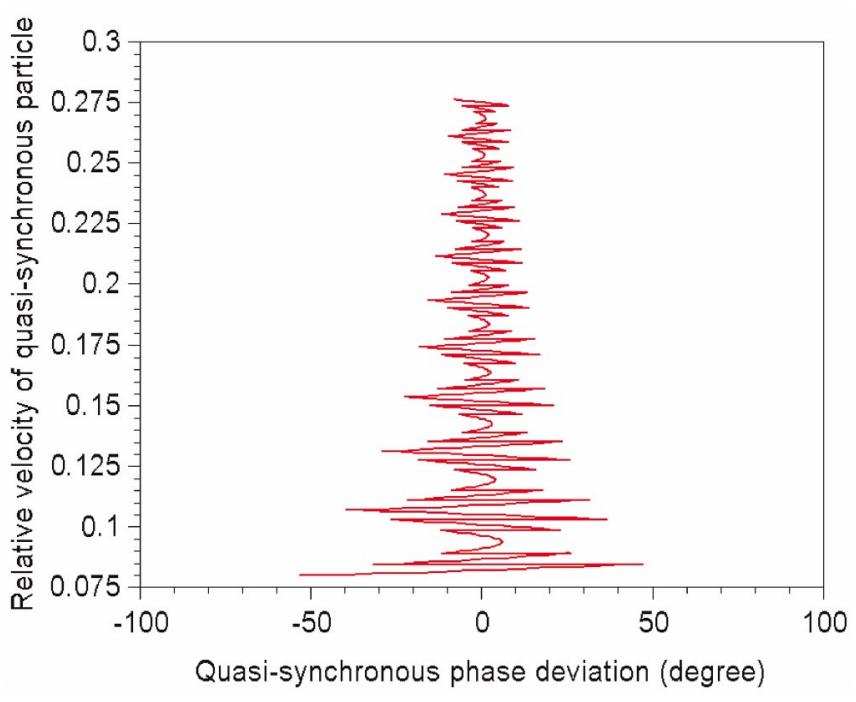

FIG. 2. (Color) Quasisynchronous particle oscillation $f(\tau)=$ $\varphi_{q s}(\tau)-\bar{\varphi}_{q s}$ relative to the average value in the linear accelerator with 10 families. 
where $A_{\mathrm{rf}}=\frac{e \lambda^{2} \beta_{\mathrm{str}}^{2}}{4 m_{0} c^{2} \gamma^{3} \beta_{q s}^{2}} \cdot k_{1} E_{1}$ is the amplitude of the $\mathrm{rf}$ defocusing factor.

Comparing the superconducting linear accelerator with the fully synchronized normal-conducting option, we can see that the sinus argument has the oscillating term. Thus, we get the alternating phase focusing component in the rf defocusing term. Let us open the sinus with the sinus argument, using the Bessel functions and retaining no terms higher than the first-order term:

$$
\begin{aligned}
\frac{d^{2} x}{d \xi^{2}} & +A_{\mathrm{rf}}\left(\frac{n_{\text {cell }}}{2}\right)^{2}\left\{\sin \bar{\varphi}_{q s}\left[J_{0}\left(\varphi_{a}\right)+\ldots\right]\right. \\
& \left.-\cos \bar{\varphi}_{q s} \cdot\left[2 J_{1}\left(\varphi_{a}\right) \sin 2 \pi \xi+\ldots\right]\right\} \cdot x=0 .
\end{aligned}
$$

The Bogolyubov-Metropolsky averaging method [5] allows us to find a common case for equation

$$
\frac{d^{2} x}{d \xi^{2}}+\left(a+2 \cdot \sum_{k} q_{k} \cos 2 \pi k \xi\right) \cdot x=0
$$

the solution:

$$
x(\xi)=A \cdot\left(1+\sum_{k} \frac{q_{k}}{2 \pi^{2} k^{2}} \cos 2 \pi k \xi\right) \cos \mu \xi
$$

with the phase advance per one focusing period:

$$
\mu_{x, y}^{2}=\frac{1}{2 \pi^{2}} \sum_{k} \frac{q_{k}^{2}}{k^{2}}+a
$$

For Eq. (12) we have

$$
\begin{aligned}
\mu_{x, y}^{2} \approx & \frac{1}{2 \pi^{2}}\left[A_{\mathrm{rf}}\left(\frac{n_{\text {cell }}}{2}\right)^{2} \cos \bar{\varphi}_{q s} \cdot J_{1}\left(\varphi_{a}\right)\right]^{2} \\
& +A_{\mathrm{rf}}\left(\frac{n_{\text {cell }}}{2}\right)^{2} \sin \bar{\varphi}_{q s} J_{0}\left(\varphi_{a}\right) .
\end{aligned}
$$

As usual, since $\sin \bar{\varphi}_{q s}<0$, the second term defocuses, but it creates the focusing term as well, which is proportional to $J_{1}\left(\varphi_{a}\right)$. Additionally, due to sliding and phase oscillating, the rf defocusing is smaller by factor $J_{0}\left(\varphi_{a}\right)$. From (16) it follows that the sliding factor can provide the beam focusing without quadrupoles, when $\mu^{2}>0$, that is

$$
\left|\sin \bar{\varphi}_{q s}\right|<\frac{A_{\mathrm{rf}} n_{\mathrm{cell}}^{2} \cos ^{2} \bar{\varphi}_{q s} J_{1}^{2}\left(\varphi_{a}\right)}{8 \pi^{2} J_{0}\left(\varphi_{a}\right)}
$$

Substituting the figures for the initial energy $W=3 \mathrm{MeV}$, $E_{1}=15 \mathrm{MV} / \mathrm{m}$, we have stable motion at quasisynchronous phase $\bar{\varphi}_{q s} \approx 2^{\circ}$, if nonsynchronism is always kept with sliding amplitude $\pm \pi / 4$. However, for higher energy, for instance $W=20 \mathrm{MeV}$, the synchronous phase should be less $\varphi_{s} \leq 1^{\circ}$. And even there the phase advance is so small that the aperture radius has to take an unacceptably big value.

Nevertheless, we can conclude that the sliding factor positively affects transverse beam stability without any significant loss in accelerating rate.

\section{THE SLOT STRUCTURE}

\section{A. Slot quadrupole focusing factor}

Exciting the quadrupole mode TE211 in the $\mathrm{H}$ resonator, we can violate the axial symmetry and create the quadrupole component between two pairs of neighboring electrodes. Figure 3 shows schematically the field picture together with the electrodes placed in the $\mathrm{H}$ resonator.

Instead of one stem with the drift tube (or the spoke) $[6,7]$ we install two stems spaced at the aperture distance and forming the slot. The focusing quadrupole field is introduced into the accelerating gaps by the slot between the stems alternatively situated in the horizontal and the vertical planes from gap to gap. The fundamental mode TE211 induces the potential between neighboring pairs of stems and creates the $\pi$ mode in the longitudinal plane with the accelerating period $\beta_{\text {str }} \lambda / 2$ and the alternative rf quadrupole field in the transverse plane with period $\beta_{\text {str }} \lambda$. We call such a cavity the slot resonator. In addition to the focusing, the slot resonator is simpler of the spoke, since it has no drift tube requiring $3 \mathrm{D}$ brazing.

We investigate the electrodynamics of this resonator by CST Microwave Studio and found that the nearest modes TE411 and TE212 are located farther than $\delta f / f=50 \%$. Figure 4 shows the $3 \mathrm{D}$ picture of the slot resonator. The rectangular and round resonators were investigated. The stem shape was optimized to get the maximum ratio between the accelerating gradient and the electrical field peak on the surface. From the same point of view, the rectangular shape of the resonator was found to be preferable.

For comparison, Figs. 5 and 6 show $E_{x, y, z}$ components of the electrical field along the spoke and slot resonators at some fixed distance from the axis $x_{0}=y_{0}=2.5 \mathrm{~mm}$ for the transverse components and on axis for the longitudinal component and for very low energy $W=3 \mathrm{MeV}$.

To obtain a complete picture, we should compare the accelerating component dependence on the radius in the case of the spoke and slot structures (Fig. 7).

You can see that in the frame of the beam radius $\sim 5 \mathrm{~mm}$ the slot resonator has some advantages. Because of the quadrupole symmetry the electrical gradient has the additional term, the slot function $G_{\text {slot }}$, with the opposite sign in the horizontal and vertical planes to satisfy the Maxwell equation $\left(\partial E_{x} / \partial x\right)+\left(\partial E_{y} / \partial y\right)+\left(\partial E_{z} / \partial z\right)=0$ :

$$
\begin{aligned}
& E_{x}=-\frac{1}{2} \frac{\partial E_{z}}{\partial z} \cdot x+G_{\text {slot }} \cdot x, \\
& E_{y}=-\frac{1}{2} \frac{\partial E_{z}}{\partial z} \cdot y-G_{\text {slot }} \cdot y .
\end{aligned}
$$

Knowing the fields $E_{x}$ at $x=x_{0}$ and $E_{y}$ at $y=y_{0}$ and subtracting one equation of system (18) from another, we can find the quadrupole component: 

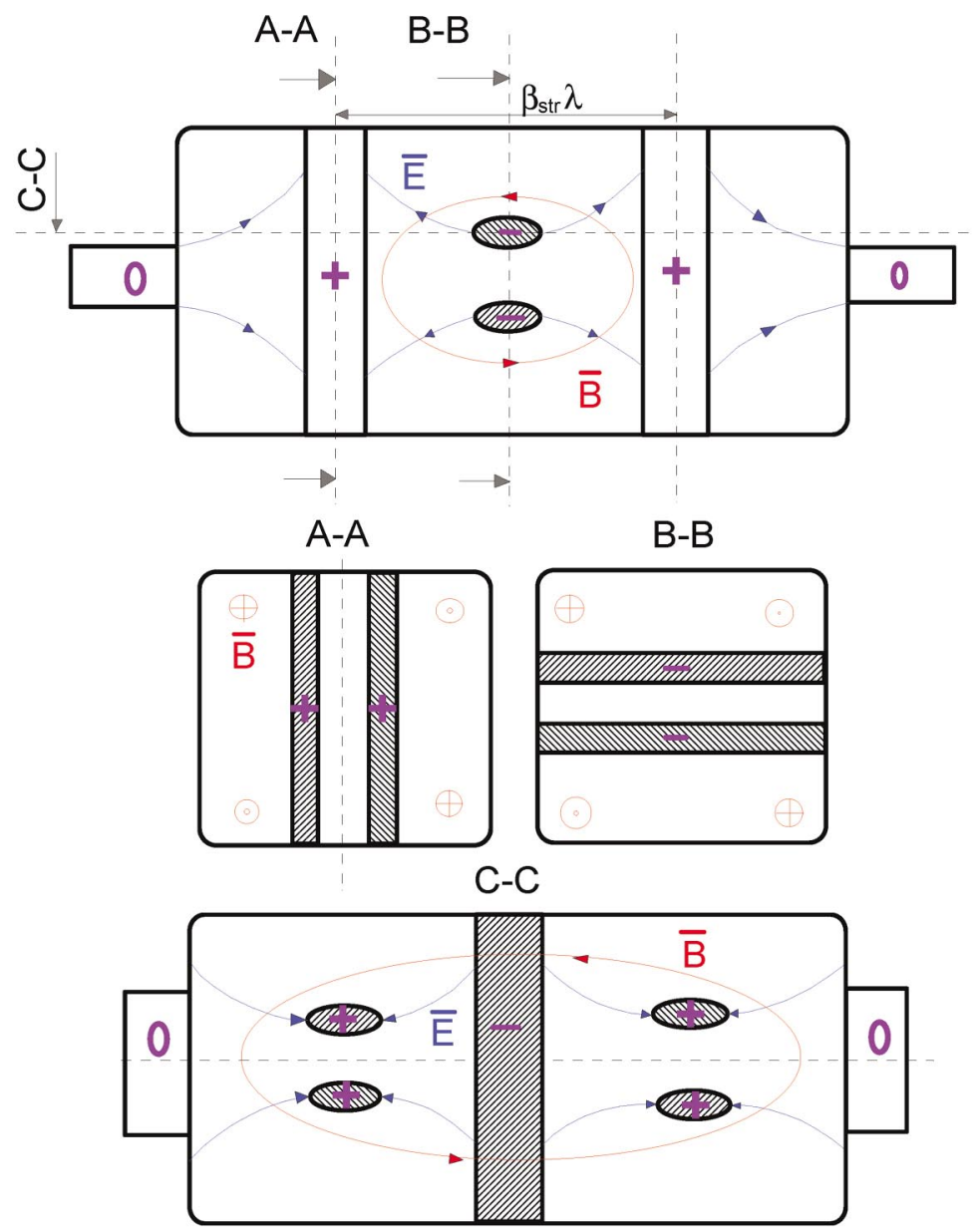

FIG. 3. (Color) Field picture in the slot resonator.

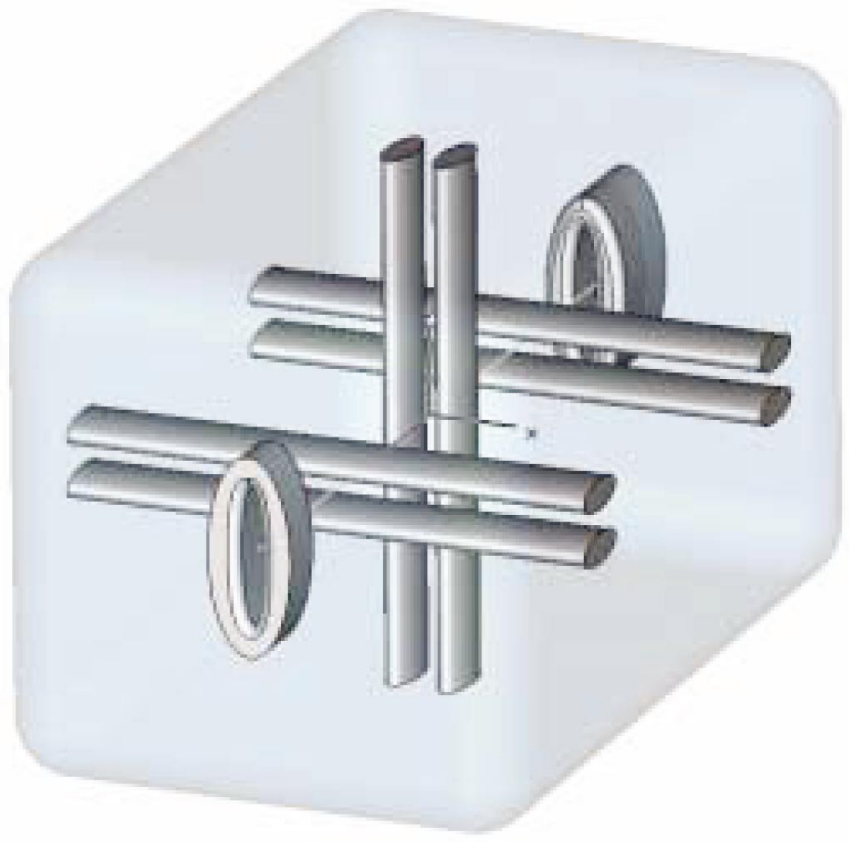

FIG. 4. (Color) Slot resonator in 3D.

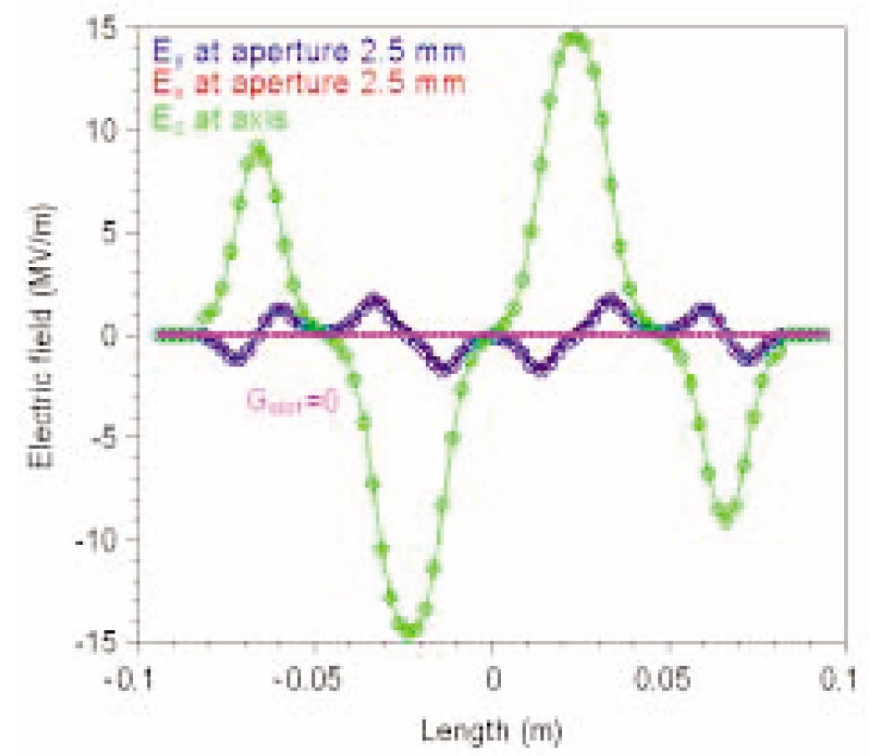

FIG. 5. (Color) $E_{x, y, z}$ components in the spoke resonator for the energy $W=3 \mathrm{MeV}$ at $E_{\text {peak }}=40 \mathrm{MV} / \mathrm{m}$. 


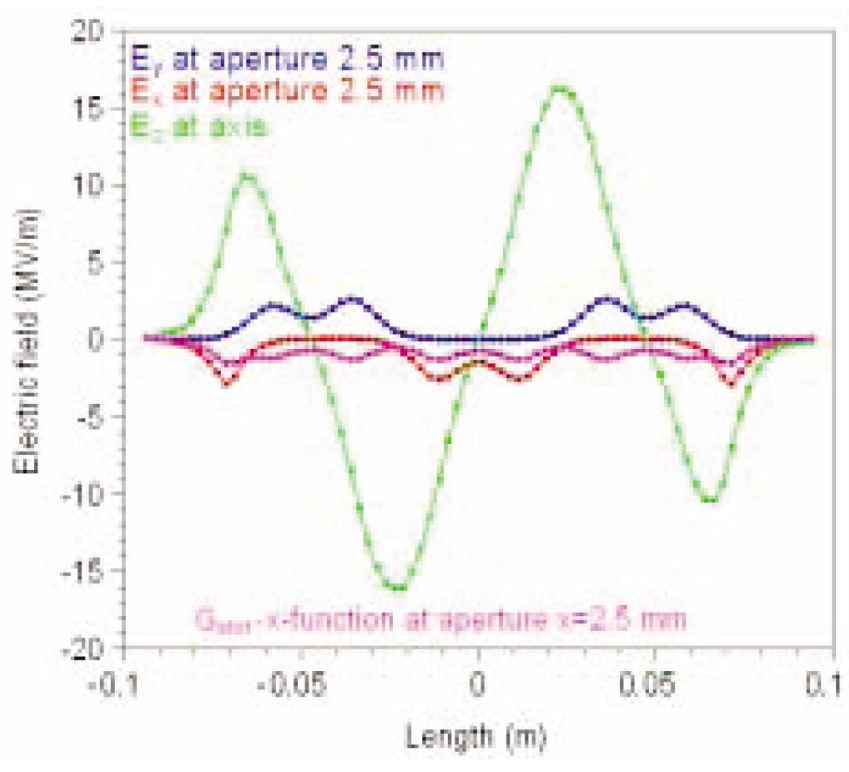

FIG. 6. (Color) $E_{x, y, z}$ components and the $G_{\text {slot }} \cdot x_{0}$ function in the slot resonator for the energy $W=3 \mathrm{MeV}$ at $E_{\text {peak }}=$ $40 \mathrm{MV} / \mathrm{m}$.

$$
G_{\text {slot }}(z, t)=\frac{1}{2}\left(\left.\frac{1}{x_{0}} \cdot E_{x}\right|_{x=x_{0}}-\left.\frac{1}{y_{0}} \cdot E_{y}\right|_{y=y_{0}}\right) \cdot \sin \left(\omega t+\varphi_{0}\right) .
$$

In the spoke resonator the quadrupole component $G_{\text {slot }}(z)$ equals zero, since $E_{x}=E_{y}$ along all cavity (see Fig. 5). Figure 6 shows the $G_{\text {slot }}(z)$ function calculated from (19) for the slot resonator. We can see the slot function can be presented as the sum of the oscillating and average (constant) components along the longitudinal coordinate. The

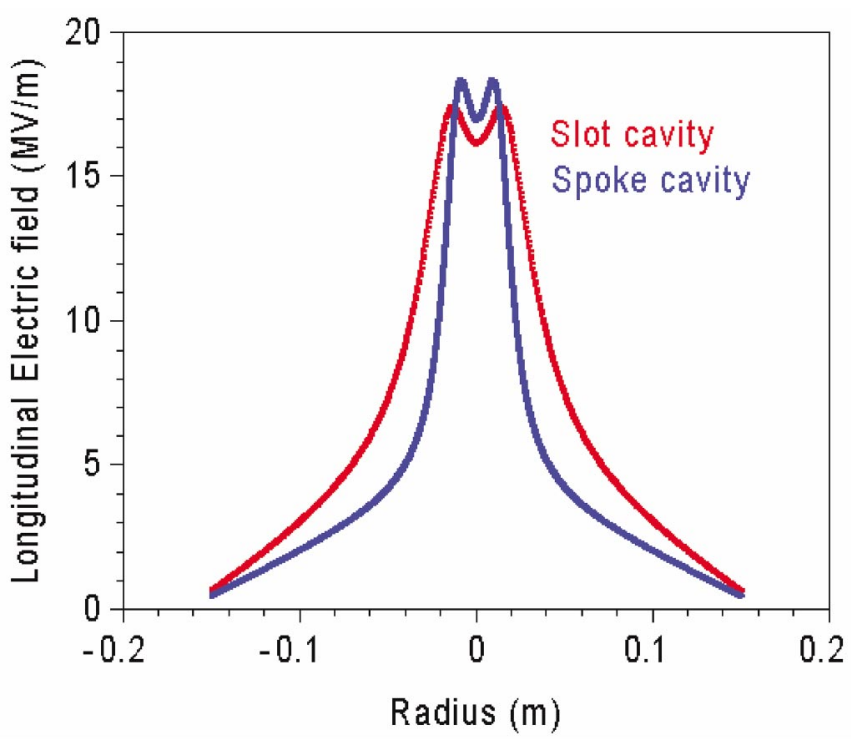

FIG. 7. (Color) The longitudinal component of the electric field in the center of the accelerating gap in the slot and spoke resonators at $E_{\text {peak }}=40 \mathrm{MV} / \mathrm{m}$. oscillating component has a shorter period by almost a factor of 4 than the cavity length, and therefore it makes an insignificant contribution to the transverse motion. Since the slots remove one of the hills in the horizontal and vertical planes (compare Figs. 5 and 6) it is reasonable to suppose that the average component is almost equal to the amplitude of the rf defocusing force:

$$
\bar{G}_{\text {slot }}=\overline{\frac{1}{2}\left(\left.\frac{1}{x_{0}} \cdot E_{x}\right|_{x=x_{0}}-\left.\frac{1}{y_{0}} \cdot E_{y}\right|_{y=y_{0}}\right)} \approx \frac{1}{2} \cdot k_{1} E_{1} .
$$

Thus, the transverse field can be presented through the sum of the fields with the alternative gradient $G_{\text {slot }}=$ $\pm \frac{1}{2} k_{1} E_{1} \sin \left(\omega t+\varphi_{0}\right)$ and the defocusing gradient $-\frac{1}{2} \cdot$ $k_{1} E_{1} \cos k_{1} z \sin \left(\omega t+\varphi_{0}\right)$. Passing on to the first traveling harmonic system, when $\omega t+\varphi_{0}=\varphi+k_{1} z$, and using the same designations for $A_{\mathrm{rf}}=\left(e \lambda^{2} \beta_{\mathrm{str}}^{2} / 4 m_{0} c^{2} \gamma^{3} \beta_{q s}^{2}\right) \cdot k_{1} E_{1}$ as in (11), the motion equation of the quasisynchronous particle $\varphi=\varphi_{q s}$ in the longitudinal coordinate with $\tau=$ $z / \beta_{\mathrm{str}} \lambda$ is

$$
\frac{d^{2} x}{d \tau^{2}}=\mp 2 A_{\mathrm{rf}} \sin \left(2 \pi \tau+\varphi_{q s}\right) \cdot x-A_{\mathrm{rf}} \sin \varphi_{q s} \cdot x .
$$

For the vertical plane the equation has the same form. You can see that the amplitudes of the slot focusing function and the defocusing function in (21) differ by factor two, since the average component of the defocusing field is $-\frac{1}{4}$. $k_{1} E_{1} \sin \varphi_{q s}$, but $\bar{G}_{\text {slot }}=\frac{1}{2} k_{1} E_{1}$.

In the case of synchronism, the quasisynchronous particle coincides with the synchronous particle, that is $\varphi_{q s}=$ $\bar{\varphi}_{s}$, and the phase advance per one focusing period with length $L_{f}=\beta_{\text {str }} \lambda$ and, hence $T=1, \xi=\tau$, is

$$
\mu_{\mathrm{slot}}=\sqrt{\frac{A_{\mathrm{rf}}^{2}}{2 \pi^{2}}+A_{\mathrm{rf}} \sin \bar{\varphi}_{s}}
$$

From the same condition, as before $\mu^{2}>0$, we can obtain the maximum synchronous phase when the motion is stable:

$$
\left|\sin \bar{\varphi}_{s}\right|<\frac{A_{\mathrm{rf}}}{2 \pi^{2}} \quad \text { or } \quad\left|\sin \bar{\varphi}_{s}\right|<\frac{e \lambda^{2} \beta_{\mathrm{str}}^{2}}{8 \pi^{2} m_{0} c^{2} \gamma^{3} \beta_{q s}^{2}} k_{1} E_{1} \text {. }
$$

\section{B. The sliding factor in the slot structure}

Now let us consider the case, when we have no synchronism in the slot structure, and the quasisynchronous particle oscillates around the average meaning $\varphi_{q s}(\tau)=$ $\bar{\varphi}_{q s}+\varphi_{a}(\tau) \cdot \sin \left(2 \pi \nu_{\mathrm{cav}} \tau\right)$ with the frequency of the $\mathrm{rf}$ shift $\nu_{\text {cav }}=\left(2 / n_{\text {cell }}\right)$. Passing on to the new coordinates system with normalized $\xi=\left(\tau / T_{\text {cav }}\right)$, where $T_{\text {cav }}=$ $n_{\text {cell }} / 2$, Eq. (21) is 


$$
\frac{d^{2} x}{d \xi^{2}}=\mp 2 A_{\mathrm{rf}}\left(\frac{n_{\text {cell }}}{2}\right)^{2} \sin \left(2 \pi \frac{n_{\text {cell }}}{2} \xi+\bar{\varphi}_{q s}+\varphi_{a} \sin 2 \pi \xi\right) \cdot x-A_{\mathrm{rf}}\left(\frac{n_{\text {cell }}}{2}\right)^{2} \sin \left(\bar{\varphi}_{q s}+\varphi_{a} \sin 2 \pi \xi\right) \cdot x .
$$

Opening the argument of the trigonometric functions and using the known relations between them and the Bessel functions, we have the equation, for instance, in the horizontal plane:

$$
\begin{aligned}
\frac{d^{2} x}{d \xi^{2}} & +2 A_{\mathrm{rf}}\left(\frac{n_{\text {cell }}}{2}\right)^{2}\left[J_{0}\left(\varphi_{a}\right) \cos \bar{\varphi}_{q s} \sin 2 \pi \frac{n_{\text {cell }}}{2} \xi+J_{0}\left(\varphi_{a}\right) \sin \bar{\varphi}_{q s} \cos 2 \pi \frac{n_{\text {cell }}}{2} \xi-2 J_{1}\left(\varphi_{a}\right) \sin \bar{\varphi}_{q s} \sin 2 \pi \frac{n_{\text {cell }}}{2} \xi \sin 2 \pi \xi\right. \\
& \left.+2 J_{1}\left(\varphi_{a}\right) \cos \bar{\varphi}_{q s} \cos 2 \pi \frac{n_{\text {cell }}}{2} \xi \sin 2 \pi \xi-J_{0}\left(\varphi_{a}\right) \sin \bar{\varphi}_{q s}+2 J_{1}\left(\varphi_{a}\right) \cos \bar{\varphi}_{q s} \sin 2 \pi \xi\right] \cdot x=0 .
\end{aligned}
$$

Then the phase advance per focusing period (one cavity length) is

$$
\mu_{\mathrm{cav}}^{2}=\frac{1}{2 \pi^{2}}\left[A_{\mathrm{rf}}\left(\frac{n_{\mathrm{cell}}}{2}\right)^{2}\right]^{2}\left[\frac{J_{0}^{2}\left(\varphi_{a}\right)}{\left(\frac{n_{\mathrm{cell}}}{2}\right)^{2}}+\frac{J_{1}^{2}\left(\varphi_{a}\right)}{\left(\frac{n_{\mathrm{cell}}}{2}-1\right)^{2}}+4 J_{1}^{2}\left(\varphi_{a}\right) \cos ^{2} \bar{\varphi}_{q s}\right]+A_{\mathrm{rf}}\left(\frac{n_{\mathrm{cell}}}{2}\right)^{2} J_{0}\left(\varphi_{a}\right) \sin \bar{\varphi}_{q s} .
$$

In our design, we consider the cavities with 4 accelerating cells $\left(n_{\text {cell }}=4\right)$, therefore the phase advance per cavity is

$$
\mu_{\mathrm{cav}}=2 \sqrt{\frac{A_{\mathrm{rf}}^{2}}{2 \pi^{2}}\left[J_{0}^{2}\left(\varphi_{a}\right)+4 J_{1}^{2}\left(\varphi_{a}\right)+16 J_{1}^{2}\left(\varphi_{a}\right) \cos ^{2} \bar{\varphi}_{q s}\right]+A_{\mathrm{rf}} J_{0}\left(\varphi_{a}\right) \sin \bar{\varphi}_{q s}}
$$

The sliding factor together with the slot quadrupole gains focusing by factor $\sqrt{\left[J_{0}^{2}\left(\varphi_{a}\right)+4 J_{1}^{2}\left(\varphi_{a}\right)+16 J_{1}^{2}\left(\varphi_{a}\right) \cos ^{2} \bar{\varphi}_{q s}\right] /\left[J_{0}\left(\varphi_{a}\right)\right]}$. Since it is bigger than the unit for any value of $\varphi_{a}$, the focusing effect is always increased.

The stability criterion, from (27), is determined by the formula:

$$
\left|\sin \bar{\varphi}_{q s}\right|<A_{\mathrm{rf}} \frac{J_{0}^{2}\left(\varphi_{a}\right)+4 J_{1}^{2}\left(\varphi_{a}\right)+16 J_{1}^{2}\left(\varphi_{a}\right) \cos ^{2} \bar{\varphi}_{q s}}{2 \pi^{2} J_{0}\left(\varphi_{a}\right)} .
$$

For $\varphi_{a}=0$, when sliding is absent, Eq. (28) coincides with (23), and formula (27) becomes (22) with the double phase advance per cavity, which is in agreement with their length ratio

$$
\mu_{\mathrm{cav}}=2 \sqrt{\frac{A_{\mathrm{rf}}^{2}}{2 \pi^{2}}+A_{\mathrm{rf}} \sin \bar{\varphi}_{q s}}=2 \mu_{\mathrm{slot}}
$$

For the case without the slot quadrupole, Eq. (27) converts into (16). Thus, Eq. (27) is the general expression, describing all the cases investigated above.

Now let us estimate the maximum possible synchronous phase for the slot structure, if nonsynchronism is always kept with sliding amplitude $\pm \pi / 4$. Substituting the figures for the initial energy $W=3 \mathrm{MeV}$, the rf frequency $f_{\text {rf }}=$ $352 \mathrm{MHz}$ and the first harmonic $E_{1}=15 \mathrm{MV} / \mathrm{m}$, the amplitude of rf defocusing factor is $A_{\mathrm{rf}} \approx 0.267$, and we are restricted by the synchronous phase $\left|\bar{\varphi}_{q s}\right| \approx 5^{\circ}$.

\section{SLOT-FINGER STRUCTURE}

\section{A. The rf fingers to obtain stable transverse motion}

Thus, even the slot focusing and the additional sliding effect together cannot provide the appropriate rf focusing channel with a reasonable aperture for the high-intensity beam.

Now let us consider the structure with additional rf electrodes installed on the extreme slots and the cavity ends as shown in Figs. 8 and 9. To minimize the influence of finger on the neighboring mode excitation the cavity has to be symmetrical relative to the cavity center. Therefore the fingers are installed symmetrically in the extreme cells only. Such a cavity is called the slot-finger resonator.

First of all, we should determine the requirements for the slot-finger resonator, which result from the beam dynamics. The equation system (9) with the rf transverse focusing field gradient $G_{\text {rf }}$ and the average slot focusing $\bar{G}_{\text {slot }}$ in coordinate $\tau=z / \beta_{\text {str }} \lambda$ is

$$
\begin{aligned}
\frac{d^{2} x}{d \tau^{2}}= & \mp \frac{e \lambda^{2} \beta_{\mathrm{str}}^{2} G_{\mathrm{rf}}(\tau)}{m_{0} c^{2} \gamma \beta_{q s}^{2}} \sin \left(2 \pi \tau+\varphi_{q s}\right) \cdot x \mp \frac{e \lambda^{2} \beta_{\mathrm{str}}^{2} \bar{G}_{\text {slot }}}{m_{0} c^{2} \gamma \beta_{q s}^{2}} \\
& \times \sin \left(2 \pi \tau+\varphi_{q s}\right) \cdot x-\frac{e \lambda^{2} \beta_{\mathrm{str}}^{2}}{4 m_{0} c^{2} \gamma^{3} \beta_{q s}^{2}} \cdot k_{1} E_{1} \sin \varphi_{q s} \cdot x, \\
\frac{d^{2} y}{d \tau^{2}}= & \pm \frac{e \lambda^{2} \beta_{\mathrm{str}}^{2} G_{\mathrm{rf}}(\tau)}{m_{0} c^{2} \gamma \beta_{q s}^{2}} \sin \left(2 \pi \tau+\varphi_{q s}\right) \cdot y \pm \frac{e \lambda^{2} \beta_{\mathrm{str}}^{2} \bar{G}_{\mathrm{slot}}}{m_{0} c^{2} \gamma \beta_{q s}^{2}} \\
& \times \sin \left(2 \pi \tau+\varphi_{q s}\right) \cdot y-\frac{e \lambda^{2} \beta_{\mathrm{str}}^{2}}{4 m_{0} c^{2} \gamma^{3} \beta_{q s}^{2}} \cdot k_{1} E_{1} \sin \varphi_{q s} \cdot y,
\end{aligned}
$$

where the slot focusing is determined by $\bar{G}_{\text {slot }}=\frac{1}{2} k_{1} E_{1}$ [see Eq. (20)]. Figure 10 shows the field distribution in the transverse and longitudinal planes calculated by CST Microwave Studio.

\section{B. The lattice of the slot-finger structure}

In order to solve equation system (30) we should decide what kind of focusing system should be applied for our 

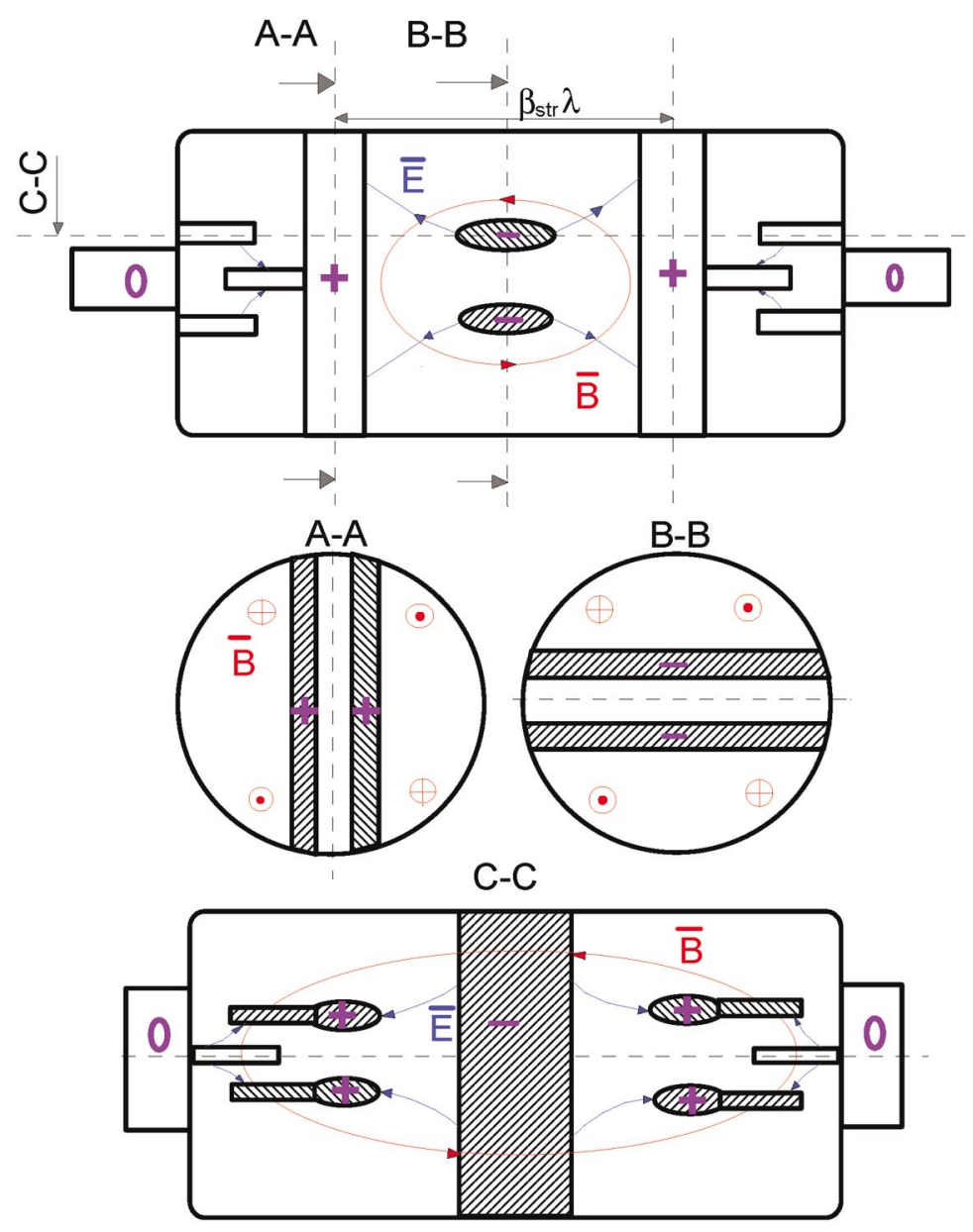

FIG. 8. (Color) Field picture in the slot-finger resonator.

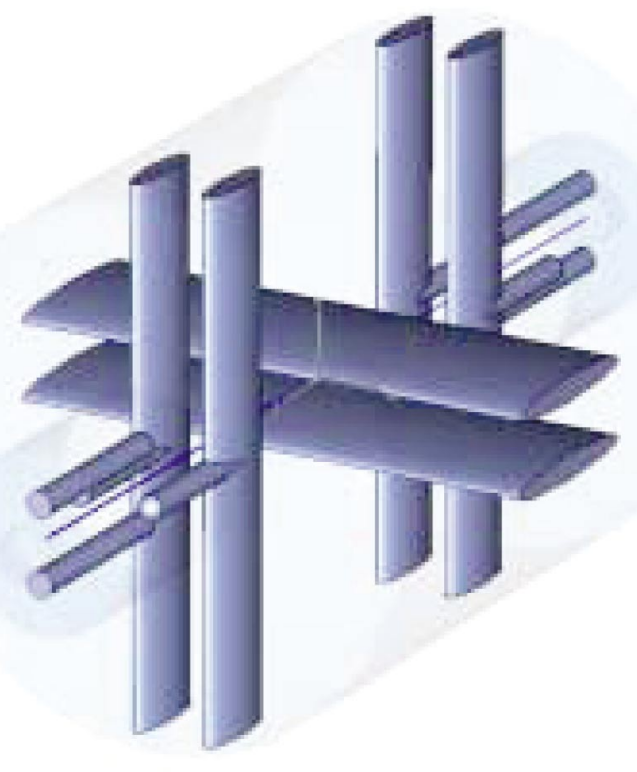

FIG. 9. (Color) The slot-finger resonator in 3D.

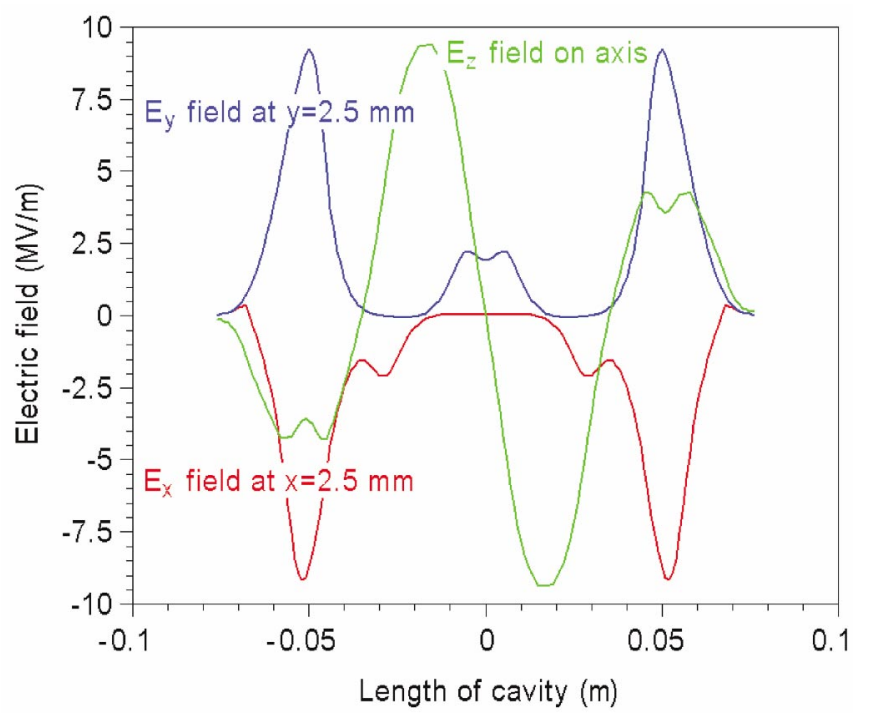

FIG. 10. (Color) Field distribution in the slot-finger resonator. 
transport channel. Figure 11 shows schematically the different lattices of the slot-finger structure. The pictures show the field, which the quasisynchronous particle "sees," that is $G_{\mathrm{rf}, \text { slot }}(z, t)=G_{\mathrm{rf} \text {,slot }}(z) \cdot \sin \omega t$.

In principle, we have only two options for the lattices based on the slot structure and shown in Figs. 11(a) and 11(b): the periodical chain of cavities and the sequence of cavities obtained by rotating every second cavity by $90^{\circ}$ around the longitudinal direction. The third option, Fig. 11(c), can only be realized in the spoke structure by the finger rotation. In first option we can produce the FOOD lattice with the periodicity of one cavity. In the second option there is the FOODDOOF lattice with the two-cavity periodicity.

Let us consider the FOOD option [Fig. 11(a)], using the matrix formalism of the thin lenses. For simplicity we take the rf finger quadrupoles only, omitting the defocusing factor and the slot quadrupoles. The accelerating gaps are represented as the drift. This approach does not pretend to achieve a very accurate calculation, but it is used for a comparable analysis of the different lattices.

Each cavity therefore has the matrix

$$
T_{x, y}=\left(\begin{array}{cc}
1 & 0 \\
\mp d_{x, y} \cos \varphi_{1} & 1
\end{array}\right)\left(\begin{array}{ll}
1 & l \\
0 & 1
\end{array}\right)\left(\begin{array}{cc}
1 & 0 \\
\pm d_{x, y} \cos \varphi_{2} & 1
\end{array}\right),
$$

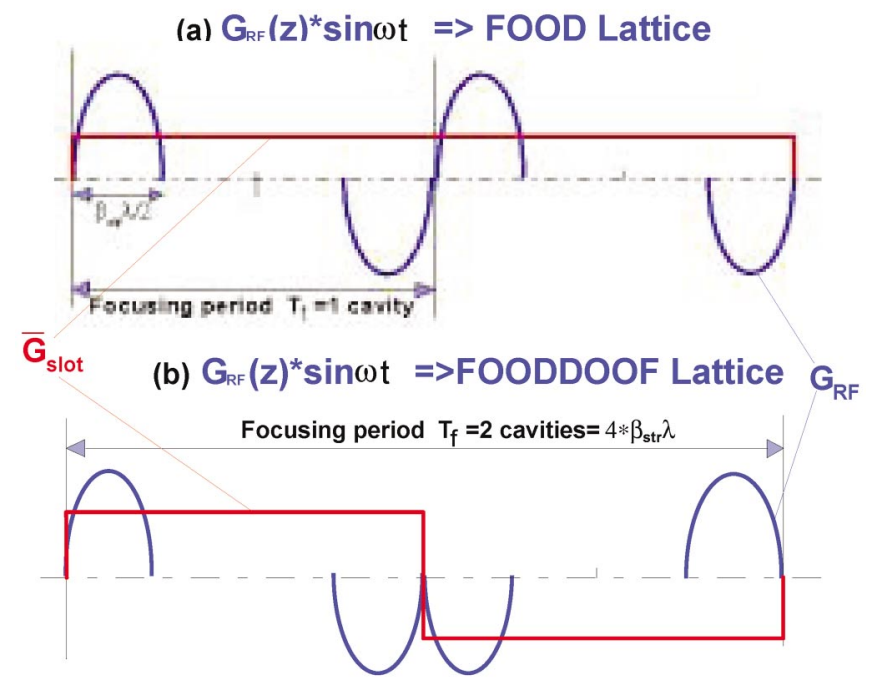

(c) $G_{R F}(z)^{*} \sin \omega t=>$ FOOFDOOD Lattice

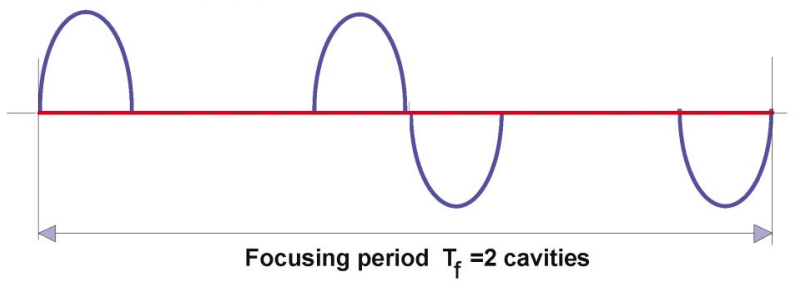

FIG. 11. (Color) Three options for the rf and the slot gradient functions. where $l$ is the distance between the centers of the rf finger quadrupoles. We assume here that the quasisynchronous particle is not synchronous and slides relative to the $\mathrm{rf}$ focusing field, passing the first and second rf finger quadrupoles with the average phase $\varphi_{1}$ and $\varphi_{2}$, respectively. The refraction angle in the $\mathrm{rf}$ finger quadrupole is designated as $d_{x, y}$ with different signs depending on the plane:

$$
d_{x, y}= \pm \frac{e G_{\mathrm{rf}}}{2 m_{0} c^{2} \gamma \beta^{2}} \frac{\sin \alpha}{\alpha} \cdot L_{\mathrm{rf}}
$$

where $\alpha=\frac{\pi}{2}\left[1-\left(\beta_{\text {str }} / \beta\right)\right]$ is the transverse transient factor, and $L_{\mathrm{rf}}$ is the rf finger quadrupole length. Multiplying the matrixes in (31), we can get the spur of the total matrix:

$$
\operatorname{Sp}\left\{M_{\text {total }}\right\}=1 \mp \frac{d_{x, y} l}{2}\left(\cos \varphi_{1}-\cos \varphi_{2}\right) .
$$

If we assume that the quasisynchronous particle executes a symmetrical oscillation around $\bar{\varphi}_{q s}$ with the amplitude $0.5 \cdot \Delta \varphi_{\text {slide }}$, that is $\varphi_{1}=-0.5 \cdot \Delta \varphi_{\text {slide }}+\bar{\varphi}_{q s}$ and $\varphi_{2}=$ $0.5 \cdot \Delta \varphi_{\text {slide }}+\bar{\varphi}_{q s}$ with definition of $\Delta \varphi_{\text {slide }}$ in (7), we have the phase advance in both planes for one focusing period:

$$
\cos \mu_{x, y}=1 \mp d_{x, y} l \sin \bar{\varphi}_{q s} \sin \frac{\Delta \varphi_{\text {slide }}}{2} .
$$

Thus, for the FOOD lattice stable motion in both planes is impossible in the case of zero gap between two neighboring cavities. In reality, we always have some distance $l_{\text {drift }}$ between the $\mathrm{F}$ and $\mathrm{D}$ quadrupoles. Then the spur of the total matrix is

$$
\begin{aligned}
\operatorname{Sp}\left\{M_{\text {total }}\right\}_{x, y}= & 1 \mp \frac{d_{x, y}}{2}\left(l+l_{\text {drift }}\right) \cdot\left(\cos \varphi_{1}-\cos \varphi_{2}\right) \\
& -\frac{l_{\text {drift }} l d_{x, y}^{2}}{2} \cos \varphi_{1} \cos \varphi_{2} .
\end{aligned}
$$

The phase advance in both planes is

$$
\begin{aligned}
\cos \mu_{x, y}= & 1 \mp d_{x, y}\left(l+l_{\text {drift }}\right) \sin \bar{\varphi}_{q s} \sin \frac{\Delta \varphi_{\text {slide }}}{2} \\
& -\frac{l_{\text {drift }} l d_{x, y}^{2}}{4}\left(\cos 2 \bar{\varphi}_{q s}+\cos \Delta \varphi_{\text {slide }}\right) .
\end{aligned}
$$

We can see the advanced phases in both planes are different due to the sliding $\left(\Delta \varphi_{\text {slide }} \neq 0\right)$, but in order to provide stable motion in both planes a high gradient in the $\mathrm{rf}$ quadrupoles is needed.

The FOODDOOF option is realized when every second cavity is rotated around the longitudinal axis in order to invert the horizontal plane into the vertical plane and the vertical plane into the horizontal plane. In that case, the total matrix of one focusing period is 


$$
\begin{aligned}
T_{x, y}= & \left(\begin{array}{cc}
1 & 0 \\
\mp d_{x, y} \cos \varphi_{1} & 1
\end{array}\right)\left(\begin{array}{ll}
1 & l \\
0 & 1
\end{array}\right)\left(\begin{array}{cc}
1 & 0 \\
\pm d_{x, y} \cos \varphi_{2} & 1
\end{array}\right) \\
& \times\left(\begin{array}{cc}
1 & 0 \\
\pm d_{x, y} \cos \varphi_{1} & 1
\end{array}\right)\left(\begin{array}{ll}
1 & l \\
0 & 1
\end{array}\right)\left(\begin{array}{cc}
1 & 0 \\
\mp d_{x, y} \cos \varphi_{2} & 1
\end{array}\right)
\end{aligned}
$$

The matrix spur is

$$
S p\left\{M_{\text {total }}\right\}_{x, y}=1-\frac{d_{x, y}^{2} l^{2}}{2}\left(\cos \varphi_{1}+\cos \varphi_{2}\right)^{2},
$$

and at symmetrical oscillation, when $\varphi_{1}=-0.5$. $\Delta \varphi_{\text {slide }}+\bar{\varphi}_{q s}$ and $\varphi_{2}=0.5 \cdot \Delta \varphi_{\text {slide }}+\bar{\varphi}_{q s}$, the phase advance in both planes is

$$
\begin{aligned}
\cos \mu_{x, y} & =1-2 d_{x, y}^{2} l^{2} \cos ^{2} \bar{\varphi}_{q s} \cos ^{2} \frac{\Delta \varphi_{\text {slide }}}{2} \text { and } \\
\mu_{x, y} & =2 d_{x, y} l \cos \bar{\varphi}_{q s} \cos \frac{\Delta \varphi_{\text {slide }}}{2}
\end{aligned}
$$

Thus, in the FOODDOOF lattice the phase advance weakly depends on the sliding factor and it can achieve the appropriate value at the lower rf gradient in comparison to the FOOD lattice.

Now let us analyze the FOODDOOF lattice in more detail. First of all, we should consider more realistic case where we have some drift $l_{\text {drift }}$ between the cavities. Omitting the calculations with the matrixes, we just present the final expression for the case with the drift:

$$
\begin{aligned}
\cos \mu_{x, y}= & 1-2 d_{x, y}^{2} l^{2} \cos ^{2} \bar{\varphi}_{q s} \cos ^{2} \frac{\Delta \varphi_{\text {slide }}}{2} \\
& -4 d_{x, y}^{2} l_{\text {drift }} l \sin \bar{\varphi}_{q s} \sin ^{2} \frac{\Delta \varphi_{\text {slide }}}{2} .
\end{aligned}
$$

It is important that the additional intercavity drift does not significantly change the phase advance, and the advanced phases in both planes remain identical.

Now we will consider how the energy growth affects the advanced phases when the refraction angle changes with the energy, that is instead of the constant $d$ we have $d_{1}, d_{2}, d_{2}, d_{3}$ and so on. In that case the total matrix of the focusing period is

$$
\begin{aligned}
T_{x, y}= & \left(\begin{array}{cc}
1 & 0 \\
\mp d_{1} \cos \varphi_{1} & 1
\end{array}\right)\left(\begin{array}{ll}
1 & l \\
0 & 1
\end{array}\right)\left(\begin{array}{cc}
1 & 0 \\
\pm d_{2} \cos \varphi_{2} & 1
\end{array}\right) \\
& \times\left(\begin{array}{cc}
1 & 0 \\
\pm d_{2} \cos \varphi_{1} & 1
\end{array}\right)\left(\begin{array}{ll}
1 & l \\
0 & 1
\end{array}\right)\left(\begin{array}{cc}
1 & 0 \\
\mp d_{3} \cos \varphi_{2} & 1
\end{array}\right)
\end{aligned}
$$

If we suppose that $d_{1}-d_{2} \approx d_{2}-d_{3}=\delta d$, we can obtain the formula for the advanced phases in both planes:

$$
\begin{aligned}
\cos \mu_{x, y}= & 1 \mp 2 l \delta d \sin \bar{\varphi}_{q s} \sin \frac{\Delta \varphi_{\text {slide }}}{2} \\
& -l^{2} \cos \bar{\varphi}_{q s} \cos \frac{\Delta \varphi_{\text {slide }}}{2}\left(d_{1} d_{2} \cos \varphi_{1}\right. \\
& \left.+d_{2} d_{3} \cos \varphi_{2}\right) .
\end{aligned}
$$

You can see that the phase advance is different in the vertical and horizontal planes due to the acceleration and changing of the refraction angle $\delta d$, and this factor is multiplied by the sliding factor. Nevertheless, comparing all parameters, the FOODDOOF lattice appears to be the best. Therefore in the following we will consider the FOODDOOF option only.

\section{Traveling wave formalism for transverse motion}

Unfortunately the matrix formalism with its discrete description does not allow all effects to be taken into account. Therefore we will pass to the traveling wave formalism. From Figs. 10 and 11(b) we can see that the transverse gradient $G_{\mathrm{rf}}(z)$ is the odd function with the amplitude $G_{\max }^{\mathrm{rf}}$ and the normalized period of two cavities $T_{f}=\left(4 \beta_{\text {str }} \lambda / \beta_{\text {str }} \lambda\right)=4$. The strongest Fourier harmonics of $G_{\text {rf }}$ are the third and the fifth.

The slot gradient function $G_{\text {slot }}$ has a meander shape with a step size of $G_{\max }^{\text {slot }}=\bar{G}_{\text {slot }}$. It is an odd function as well, and it has the same period $T=4$, but the lowest harmonics are the first and third.

Later we will show that it is reasonable to retain only the nearest harmonics for the $\mathrm{rf}$ focusing gradient $G_{3}^{\mathrm{rf}}=$ $G_{\max }^{\mathrm{rf}} F_{3}^{\mathrm{rf}} \sin \frac{3}{4} 2 \pi \tau, \quad G_{5}^{\mathrm{rf}}=G_{\max }^{\mathrm{rf}} F_{5}^{\mathrm{rf}} \sin \frac{5}{4} 2 \pi \tau \quad$ and $\quad$ for the slot gradient $G_{1}^{\text {slot }}=G_{\max }^{\text {slot }} F_{1}^{\text {slot }} \sin \frac{1}{4} 2 \pi \tau, G_{3}^{\text {slot }}=$ $G_{\max }^{\text {slot }} F_{3}^{\text {slot }} \sin \frac{3}{4} 2 \pi \tau$, where $F_{n}^{\text {rf }}, F_{n}^{\text {slot }}$ are the form factors of field distribution for the $n$th harmonic:

$$
\begin{aligned}
F_{n}^{\mathrm{rf}} & =\frac{1}{G_{\max }^{\mathrm{rf}}} \int_{0}^{2} G_{\mathrm{rf}}(\tau) \sin \frac{1}{4} 2 \pi n \tau d \tau, \\
F_{n}^{\text {slot }} & =\frac{1}{G_{\max }^{\mathrm{slot}}} \int_{0}^{2} \bar{G}_{\text {slot }}(\tau) \sin \frac{1}{4} 2 \pi n \tau d \tau .
\end{aligned}
$$

In particular, for the "sinus" approximation of the electrical field distribution along the focusing cells $G_{\text {rf }}(\tau)=$ $G_{\max }^{\mathrm{rf}} \sin \frac{1}{4} 2 \pi \tau$ and the meander shape of the slot function $\bar{G}_{\text {slot }}(\tau)$, the nearest harmonics have the values $F_{3}^{\mathrm{rf}}=F_{5}^{\mathrm{rf}}=$ $(\sqrt{2} / \pi)$ and $F_{1}^{\text {slot }}=\frac{4}{\pi}=3 \cdot F_{3}^{\text {slot }}$. Using the same designation as before together with the new designation for $\quad Q_{\mathrm{rf}}=\left(e \lambda^{2} \beta_{\mathrm{str}}^{2} G_{\max }^{\mathrm{rf}} / m_{0} c^{2} \gamma \beta_{q s}^{2}\right) \quad$ and $\quad Q_{\text {slot }}=$ $\left(e \lambda^{2} \beta_{\mathrm{str}}^{2} / m_{0} c^{2} \gamma \beta_{q S}^{2}\right) \cdot \frac{1}{2} k_{1} E_{1}$, we have the motion equations: 


$$
\begin{aligned}
\frac{d^{2} x}{d \tau^{2}}= & \mp Q_{\mathrm{rf}} \cdot x \cdot \sin \left(2 \pi \tau+\varphi_{q s}\right) \cdot\left[F_{3}^{\mathrm{rf}} \sin \left(\frac{3}{4} 2 \pi \tau\right)+F_{5}^{\mathrm{rf}} \sin \left(\frac{5}{4} 2 \pi \tau\right)\right] \mp Q_{\text {slot }} \cdot x \cdot \sin \left(2 \pi \tau+\varphi_{q s}\right) F_{1}^{\text {slot }} \sin \frac{1}{4} 2 \pi \tau \\
& \mp Q_{\text {slot }} \cdot x \cdot \sin \left(2 \pi \tau+\varphi_{q s}\right) F_{3}^{\text {slot }} \sin \frac{3}{4} 2 \pi \tau-A_{\mathrm{rf}} \cdot \sin \varphi_{q s} \cdot x \\
\frac{d^{2} y}{d \tau^{2}}= & \pm Q_{\mathrm{rf}} \cdot y \cdot \sin \left(2 \pi \tau+\varphi_{q s}\right) \cdot\left[F_{3}^{\mathrm{rf}} \sin \left(\frac{3}{4} 2 \pi \tau\right)+F_{5}^{\mathrm{rf}} \sin \left(\frac{5}{4} 2 \pi \tau\right)\right] \pm Q_{\text {slot }} \cdot y \cdot \sin \left(2 \pi \tau+\varphi_{q s}\right) F_{1}^{\text {slot }} \sin \frac{1}{4} 2 \pi \tau \\
& \pm Q_{\text {slot }} \cdot y \cdot \sin \left(2 \pi \tau+\varphi_{q s}\right) F_{3}^{\text {slot }} \sin \frac{3}{4} 2 \pi \tau-A_{\mathrm{rf}} \cdot \sin \varphi_{q s} \cdot y
\end{aligned}
$$

Here and in the following we should remember from the previous consideration that the slot focusing amplitude is double the defocusing amplitude $Q_{\text {slot }}=2 A_{\mathrm{rf}}$. Later for compactness we will write the equation for one plane, for instance, the horizontal plane, taking into account the fact that they have a similar view in the vertical plane.

You can see that both harmonics $F_{3}^{\mathrm{rf}}$ and $F_{5}^{\mathrm{rf}}$ together with the space periodic term $\sin 2 \pi \tau$ give the slow harmonics $(\sqrt{2} / 2 \pi) \cos \left(\frac{1}{4} 2 \pi \tau+\varphi_{q s}\right) \quad$ and $\quad(\sqrt{2} / 2 \pi) \cos \left(\frac{1}{4} 2 \pi \tau-\right.$ $\left.\varphi_{q s}\right)$. Passing on to the new coordinate system normalized by the focusing period $d \tau=T_{f} d s$, and retaining the slow harmonics only, we have

$$
\begin{aligned}
\frac{d^{2} x}{d s^{2}}= & \mp T_{f}^{2} \frac{\sqrt{2}}{\pi} Q_{\mathrm{rf}} \cos \varphi_{q s} \cos (2 \pi s) \cdot x \\
& \mp T_{f}^{2} \frac{2}{3 \pi} Q_{\mathrm{slot}} \cos \left(2 \pi s+\varphi_{q s}\right) \cdot x-T_{f}^{2} A_{\mathrm{rf}} \cdot \sin \varphi_{q s} \cdot x
\end{aligned}
$$

Using (15) and taking into account a small value of the synchronous phase, when $\sin ^{2} \varphi_{q s} \ll \cos ^{2} \varphi_{q s}$, the phase advance per focusing period (for the FOODDOOF lattice $\left.T_{f}=4\right)$ is

$$
\mu_{x, y}^{2} \approx \frac{1}{2 \pi^{2}}\left(\frac{\sqrt{2} Q_{\mathrm{rf}}}{2 \pi}+\frac{Q_{\text {slot }}}{3 \pi}\right)^{2} T_{f}^{4} \cos ^{2} \varphi_{q s}+T_{f}^{2} A_{\mathrm{rf}} \sin \varphi_{q s} .
$$

Since the function $Q_{\mathrm{rf}}$ is determined by the electrical gradient and it is much bigger than $(1 / 2)\left(\partial E_{z} / \partial z\right)$, we can provide the required phase advance value. The contribution of the slot function to the total focusing effect is determined by the ratio between the advanced phase with and without the slot term. In spite of the small ratio between $Q_{\text {slot }} / Q_{\text {rf }}$ the slot focusing contribution can achieve a significant value $\sim(15-20) \%$.

To test our traveling wave approximation (45) omitting fast harmonics we have integrated both systems (44) and (45) and compare the results (see Fig. 12). You can see that the difference is negligible. In the following we shall use the system (45).

\section{Longitudinal-transverse coupling resonance}

Now we would like to consider the resonance due to the coupling between the longitudinal and transverse motions.
Following [4] the oscillation of an arbitrary particle can be represented as the oscillation relative to the quasisynchronous particle with amplitude $\Delta \psi$ and the oscillation of the quasisynchronous particle itself relative to the average phase $\bar{\varphi}_{q s}$

$$
\varphi(s)=\Delta \psi \cdot \cos \left(\Omega_{L} \cdot s\right)+\varphi_{q s}(s)
$$

where the phase oscillation $\varphi_{q s}(s)$ is determined by (10) and the longitudinal frequency is

$$
\Omega_{L}^{2}=-\frac{\pi e E_{1} \sin \bar{\varphi}_{q s} \lambda \beta_{\mathrm{str}}^{2} J_{0}\left(\varphi_{a}\right) T_{f}^{2}}{m_{0} c^{2} \gamma^{3} \beta_{q s}^{3}} .
$$

In the considered energy range (3-50) $\mathrm{MeV}$ the longitudinal frequency changes $\left(\Omega_{L} / 2 \pi\right) \approx(0.2-0.05)$, and it can be regarded as a slowly changing parameter. At the same time, the advance phase in transverse planes is modulated by the longitudinal frequency, and it can cause parametric resonance in the wide range.

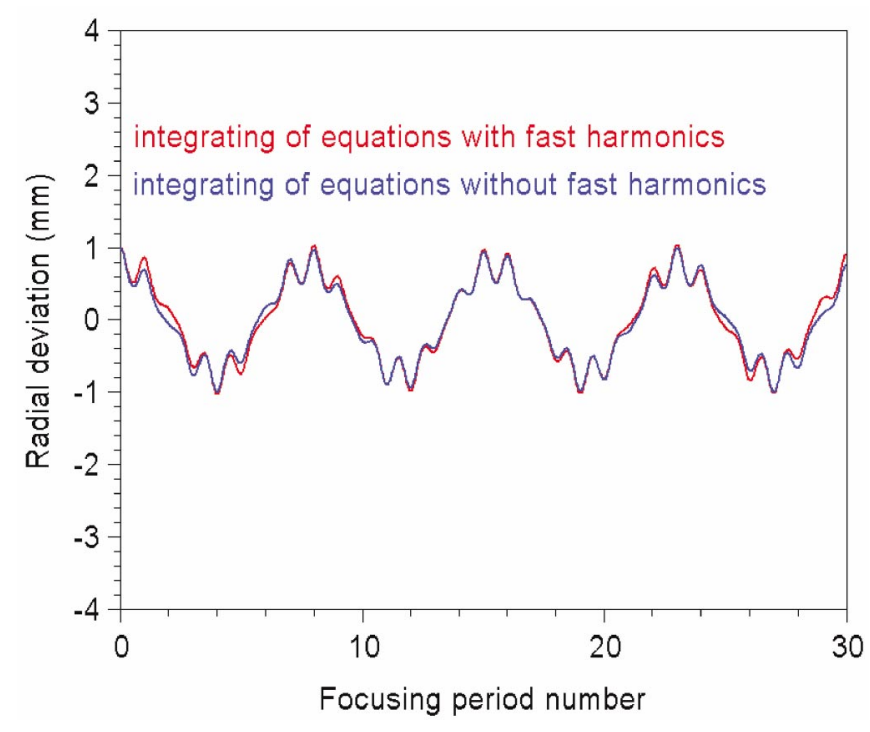

FIG. 12. (Color) Comparison of integrating results with (44) and without (45) fast harmonics in transverse gradient. 
Designating

$$
\begin{aligned}
\mu_{0}^{2} & \approx \frac{1}{2 \pi^{2}}\left(\frac{\sqrt{2} Q_{\mathrm{rf}}}{2 \pi}+\frac{Q_{\text {slot }}}{3 \pi}\right)^{2} T_{f}^{4} \cos ^{2} \varphi_{\mathrm{qs}} \quad \text { and } \\
b & =-T_{f}^{2} A_{\mathrm{rf}} \sin \varphi_{q s}>0,
\end{aligned}
$$

Equation (46) can be represented as

$$
\mu_{x, y}^{2} \approx \mu_{0}^{2}-b .
$$

Substituting (47) into (46), we have for the arbitrary particle with initial deviation from the quasisynchronous particle $\Delta \psi$ the phase advance

$$
\mu_{x, y}^{2} \approx \mu_{0}^{2}-b-\left(2 \mu_{0}^{2} \tan \varphi_{q s}+b \cot \varphi_{q s}\right) \cdot \Delta \psi \cos \Omega_{L} s .
$$

In the slot-finger structure the accelerating gradient is limited by the required focusing rf quadrupole. Therefore it is reasonable to work in the variables $\mu_{0}$ and $\Omega_{L}$. Comparing (48) and (49) we see that the defocusing factor is half of the longitudinal frequency square $b=\left(\Omega_{L}^{2} / 2\right)$. Later we will show that the sliding factor gives the Bessel coefficient in the defocusing factor similarly to the longitudinal frequency. The averaged motion equation is described by the equation:

$$
\begin{aligned}
\frac{d^{2} x}{d s^{2}}+ & {\left[\mu_{0}^{2}-\frac{\Omega_{L}^{2}}{2}-\left(2 \mu_{0}^{2} \cdot \tan \varphi_{q s}\right.\right.} \\
& \left.\left.+\frac{\Omega_{L}^{2}}{2} \cot \varphi_{q s}\right) \Delta \psi \cdot \cos \Omega_{L} s\right] \cdot x=0 .
\end{aligned}
$$

Thus, due to the longitudinal oscillation, the radial tune is modulated with amplitude $\psi_{m}=\Delta \psi \cdot\left[2 \mu_{0}^{2} \tan \varphi_{q s}+\right.$ $\left.\left(\Omega_{L}^{2} / 2\right) \cot \varphi_{q s}\right]$.

From the parametric resonance theory we have the $n$th order parametric resonance in the case of

$$
2 \sqrt{\left(\frac{\mu_{0}}{\Omega_{L}}\right)^{2}-\frac{1}{2}}=n, \quad(n=1,2,3, \ldots) .
$$

Therefore in accordance with [5] the first resonance width is

$$
\begin{aligned}
& 4\left(\mu_{0}^{2}-\frac{\Omega_{L}^{2}}{2}\right)-2\left(2 \mu_{0}^{2} \cdot\left|\tan \varphi_{q s}\right|+\frac{\Omega_{L}^{2}}{2}\left|\cot \varphi_{q s}\right|\right) \Delta \psi<\Omega_{L}^{2}, \\
& \Omega_{\mathrm{L}}^{2}<4\left(\mu_{0}^{2}-\frac{\Omega_{L}^{2}}{2}\right)+2\left(2 \mu_{0}^{2} \cdot\left|\tan \varphi_{\mathrm{qs}}\right|+\frac{\Omega_{\mathrm{L}}^{2}}{2}\left|\cot \varphi_{\mathrm{qs}}\right|\right) \Delta \psi .
\end{aligned}
$$

Since the longitudinal frequency is always smaller than the radial frequency the right condition only has to be checked:

$$
4\left(\mu_{0}^{2}-\frac{\Omega_{L}^{2}}{2}\right)-2\left(2 \mu_{0}^{2} \cdot\left|\tan \varphi_{q s}\right|+\frac{\Omega_{L}^{2}}{2}\left|\cot \varphi_{q s}\right|\right) \Delta \psi>\Omega_{L}^{2} .
$$

Opening and grouping, we have the upper boundary for the maximum possible value of the longitudinal frequency, when the first-order resonance is never crossed:

$$
\frac{\Omega_{L}}{2 \mu_{0}}<\left|\tan \varphi_{q s}\right| \sqrt{\frac{\left|\cot \varphi_{q s}\right|-\Delta \psi}{3\left|\tan \varphi_{q s}\right|+\Delta \psi}} .
$$

For the second order resonance, when $n=2$, we have from (53) $\left(\Omega_{L} / \mu_{0}\right)=\sqrt{2 / 3}$, and the resonance width is

$$
\begin{gathered}
\mu_{0}^{2}-\frac{5\left(2 \mu_{0}^{2} \cdot\left|\tan \varphi_{q s}\right|+\frac{\Omega_{L}^{2}}{2}\left|\cot \varphi_{q s}\right|\right)^{2} \Delta \psi^{2}}{12\left(\mu_{0}^{2}-\frac{\Omega_{L}^{2}}{2}\right)}<\frac{3}{2} \Omega_{L}^{2}<\mu_{0}^{2} \\
+\frac{\left(2 \mu_{0}^{2} \cdot\left|\tan \varphi_{q s}\right|+\frac{\Omega_{L}^{2}}{2}\left|\cot \varphi_{q s}\right|\right)^{2} \Delta \psi^{2}}{12\left(\mu_{0}^{2}-\frac{\Omega_{L}^{2}}{2}\right)}
\end{gathered}
$$

So, in the case where the longitudinal frequency is below the left boundary, we have no crossing with the second order resonance. Let us substitute the working parameters of the considered linear accelerator option with $\varphi_{q s}=$ $-20^{\circ}$ and the fields shown in Fig. 10. Since the longitudinal frequency decreases faster than the radial frequency, we use the initial parameters of the linear accelerator. For the accelerating rate $W=2.5 \mathrm{MeV} / \mathrm{m}$, the quasisynchronous phase $\varphi_{q s}=-20^{\circ}$ and the bunch length $30^{\circ}$ the longitudinal and radial frequencies are $\mu_{0} \approx 1.5, \Omega_{L} \approx$ 1.2 , and $\Delta \psi \approx 15^{\circ}$. From (56) it follows that the first-order resonance is not crossed, but the second order resonance (57) can be reached very easily. But the second order resonance width is relatively small and its influence can be negligible under fast crossing.

\section{E. How to minimize the influence of longitudinal oscil- lation on transverse motion}

Even under nonresonance conditions, the instantaneous transverse frequency can be unacceptably small during the long half-wave of longitudinal oscillation:

$$
\mu_{\text {inst }}=\sqrt{\mu_{0}^{2}-\frac{\Omega_{L}^{2}}{2}-\left(2 \mu_{0}^{2} \cdot \tan \varphi_{q s}+\frac{\Omega_{L}^{2}}{2} \cot \varphi_{q s}\right) \Delta \psi \cdot \cos \Omega_{L} s}
$$

To minimize this effect we shift the finger center in both rf finger quadrupoles by $\delta \varphi_{\text {shift }}$ to provide a smaller phase advance between the first rf finger quadrupole and the first accelerating cell and a bigger phase advance between the second accelerating cell and the second rf finger quadrupole. 
Then the amplitude of radial tune oscillation is

$$
\psi_{m}=\Delta \psi \cdot\left[2 \mu_{0}^{2} \tan \left(\varphi_{q s}+\delta \varphi_{\text {shift }}\right)+\frac{\Omega_{L}^{2}}{2} \cot \varphi_{q s}\right]
$$

Now equating the expression in parenthesis to zero, we can find the phase shift $\delta \varphi_{\text {shift }}$, which in the first-order approximation provides the amplitude of the radial tune oscillation equal to zero:

$$
\delta \varphi_{\text {shift }}=-\arctan \left(\frac{\Omega_{L}^{2}}{4 \mu_{0}^{2}} \cot \varphi_{q s}\right)-\varphi_{q s}
$$

Fulfilling this ratio, we force any particle to oscillate around $\varphi_{q s}$ in the longitudinal plane and around $\varphi_{q s}+$ $\delta \varphi_{\text {shift }}$ in the transverse plane. Thus, the growth of the defocusing factor for the particle located in the bunch head is compensated by a growth of $\mathrm{rf}$ focusing. And, on the contrary, for the particle located in the tail of the bunch the decrease of $\mathrm{rf}$ focusing is compensated by the weaker defocusing. For the considered linear accelerator option with $\varphi_{q s}=-20^{\circ}, \mu_{0} \approx 1.5, \Omega_{L} \approx 1.2$, and $\Delta \psi \approx 15^{\circ}$ the phase shift has to be $\sim+40^{\circ}$. This means the phase shift is almost symmetrical relative to the maximum of cosines. Actually it is not reasonable to shift more than $40^{\circ}$, since this leads to a decrease of rf focusing.

Taking into account the phase shift, the expression for $\mu_{0}$ is updated

$$
\mu_{0}^{2} \approx \frac{1}{2 \pi^{2}}\left[\frac{\sqrt{2} Q_{\mathrm{rf}}}{2 \pi} \cos \left(\varphi_{\mathrm{qs}}+\delta \varphi_{\mathrm{shift}}\right)+\frac{Q_{\text {slot }}}{3 \pi} \cos \varphi_{\mathrm{qs}}\right]^{2} T_{f}^{4}
$$

and the defocusing factor remains the same.

\section{F. The sliding factor in the slot-finger structure}

Now let us consider the influence of the nonsynchronism in the slot-finger structure when the quasisynchronous particle oscillates relative to the accelerating field with the phase $\varphi_{q s}(\tau)=\bar{\varphi}_{q s}+\varphi_{a}(\tau) \cdot \sin \left(2 \pi \nu_{\text {cav }} \tau\right)$ and relative to the rf focusing field with the phase $\varphi_{q s}(\tau)+$ $\delta \varphi_{\text {shift }}=\bar{\varphi}_{q s}+\delta \varphi_{\text {shift }}+\varphi_{a}(\tau) \cdot \sin \left(2 \pi \nu_{\text {cav }} \tau\right)$.

Substituting the latter in equation system (45), we have

$$
\begin{aligned}
\frac{d^{2} x}{d s^{2}}= & \mp T_{f}^{2} \frac{\sqrt{2}}{\pi} Q_{\mathrm{rf}} \cos \left(\bar{\varphi}_{q s}+\delta \varphi_{\text {shift }}+\varphi_{a} \sin 2 \pi \frac{T_{f}}{T_{\text {cav }}} s\right) \\
& \times \cos (2 \pi s) \cdot x \mp T_{f}^{2} \frac{2}{3 \pi} Q_{\text {slot }} \\
& \times \cos \left(2 \pi s+\bar{\varphi}_{q s}+\varphi_{a} \sin 2 \pi \frac{T_{f}}{T_{\text {cav }}} s\right) \cdot x-T_{f}^{2} A_{\mathrm{rf}} . \\
& \times \sin \left(\bar{\varphi}_{q s}+\varphi_{a} \sin 2 \pi \frac{T_{f}}{T_{\text {cav }}} s\right) \cdot x .
\end{aligned}
$$

For the vertical plane the equation is the same. Opening the argument of the trigonometric functions and using known relations between them and the Bessel functions for the lowest harmonics (not higher than $\sin \left[2 \pi\left(T_{f} / T_{\text {cav }}\right)\right] s$ ), we have the equations, for instance, in the horizontal plane:

$$
\begin{aligned}
\frac{d^{2} x}{d s^{2}}= & -T_{f}^{2} \frac{\sqrt{2}}{\pi} Q_{\mathrm{rf}}\left[J_{0}\left(\varphi_{a}\right) \cos \left(\bar{\varphi}_{q s}+\delta \varphi_{\text {shift }}\right)-2 J_{1}\left(\varphi_{a}\right) \sin \left(\bar{\varphi}_{q s}+\delta \varphi_{\text {shift }}\right) \sin 2 \pi \frac{T_{f}}{T_{\text {cav }}} s\right] \cos (2 \pi s) \cdot x \\
& -T_{f}^{2} \frac{2}{3 \pi} Q_{\text {slot }}\left[J_{0}\left(\varphi_{a}\right) \cos \bar{\varphi}_{q s}-2 J_{1}\left(\varphi_{a}\right) \sin \bar{\varphi}_{q s} \sin 2 \pi \frac{T_{f}}{T_{\text {cav }}} s\right] \cos (2 \pi s) \cdot x+T_{f}^{2} \frac{2}{3 \pi} Q_{\text {slot }}\left[J_{0}\left(\varphi_{a}\right) \sin \bar{\varphi}_{q s}\right. \\
& \left.+2 J_{1}\left(\varphi_{a}\right) \cos \bar{\varphi}_{q s} \sin 2 \pi \frac{T_{f}}{T_{\text {cav }}} s\right] \sin (2 \pi s) \cdot x-T_{f}^{2} A_{\mathrm{rf}} \cdot\left[J_{0}\left(\varphi_{a}\right) \sin \bar{\varphi}_{q s}+2 J_{1}\left(\varphi_{a}\right) \cos \bar{\varphi}_{q s} \sin 2 \pi \frac{T_{f}}{T_{\text {cav }}} s\right] \cdot x
\end{aligned}
$$

In our design, we consider the linear accelerator with the ratio $\left(T_{f} / T_{\text {cav }}\right)=2$, therefore the phase advance is

$$
\begin{aligned}
\mu_{x, y}^{2} \approx & \frac{1}{2 \pi^{2}}\left(\frac{\sqrt{2} Q_{\mathrm{rf}}}{2 \pi} J_{0}\left(\varphi_{a}\right) \cos \left(\bar{\varphi}_{q s}+\delta \varphi_{\text {shift }}\right)+\frac{Q_{\text {slot }}}{3 \pi} \cos \bar{\varphi}_{q s}\left[J_{0}\left(\varphi_{a}\right)-J_{1}\left(\varphi_{a}\right)\right]\right)^{2} T_{f}^{4}+\frac{1}{2 \pi^{2}}\left(\frac { \sqrt { 2 } Q _ { \mathrm { rf } } } { 2 \pi } J _ { 1 } ( \varphi _ { a } ) \operatorname { s i n } \left(\bar{\varphi}_{q s}\right.\right. \\
& \left.\left.+\delta \varphi_{\text {shift }}\right)+\frac{Q_{\text {slot }}}{3 \pi} \sin \bar{\varphi}_{q s}\left[J_{0}\left(\varphi_{a}\right)+J_{1}\left(\varphi_{a}\right)\right]\right)^{2} T_{f}^{4}+T_{f}^{2} A_{\mathrm{rf}} J_{0}\left(\varphi_{a}\right) \sin \bar{\varphi}_{q s} .
\end{aligned}
$$

Taking into account the symmetrical phase shift $\bar{\varphi}_{q s}+$ $\delta \varphi_{\text {shift }} \approx-\bar{\varphi}_{q s}$, the small value of the synchronous phase $\left(\sin ^{2} \bar{\varphi}_{q s} \ll \cos ^{2} \bar{\varphi}_{q s}\right)$ and the ratio $J_{0}\left(\varphi_{a}\right) \gg J_{1}\left(\varphi_{a}\right)$, the phase advance per cavity is

$$
\begin{aligned}
\mu_{x, y}^{2} \approx & \frac{1}{2 \pi^{2}}\left(\frac{\sqrt{2} Q_{\mathrm{rf}}}{2 \pi}+\frac{Q_{\text {slot }}}{3 \pi}\right)^{2} T_{f}^{4} J_{0}^{2}\left(\varphi_{a}\right) \cos ^{2}\left(\bar{\varphi}_{q s}+\delta \varphi_{\text {shift }}\right) \\
& +T_{f}^{2} A_{\mathrm{rf}} J_{0}\left(\varphi_{a}\right) \sin \bar{\varphi}_{q s} .
\end{aligned}
$$

From this expression you can see that due to the sliding the rf focusing amplitude becomes weaker by factor $J_{0}\left(\varphi_{a}\right)$ and the defocusing factor decreases by the same factor.

\section{G. The "family- structure" resonances}

To make the linear accelerator cheaper the cavities are grouped in families, and the cavities belonging to one family have identical geometry. From the cost and the radiotechnical tuning point of view this method is reasonable. But we should expect that such simplification could 
affect the beam dynamics. In a previous paper [4], the longitudinal beam dynamics was investigated in the linear accelerator with the family grouping.

In transverse motion due to the family grouping, the focusing period and the advanced phase have got a periodic component. Since all cavities are identical in the family, in the first cavities the structure velocity has to be higher than the bunch velocity and in the last cavities we have opposite situation. Thus, the focusing is modulated. Figure 13 shows the instantaneous phase advance in both planes in the option with 6 cavities in one family. You can see that it is modulated with periodicity $T_{f}=6$ cavities (or 3 focusing periods). From the parametric resonance theory it follows that in order to avoid the "family-structure" resonance the phase advance has to be $\mu_{x, y}<\left(2 \pi / 2 T_{f}\right)$. For the option under consideration with 6 cavities in one family the phase advance is restricted by a value of $60^{\circ}$ per focusing period (or $30^{\circ}$ per cavity) in both planes. Because of the acceleration the phase advance in the vertical and horizontal planes is different [Eq. (42)]. However, this difference decreases with energy.

Taking into account that the space charge shift is expected, we should take a smaller number of cavities in the family at least for initial acceleration, for instance 4 cavities in one family. Figure 14 shows the effect of the "family-structure" resonance crossing. We have tracked the particle in the 3D field in the accelerator with 4 cavities in the family. From the figure you can see, in the case where the phase advance is near to $90^{\circ}$, that the oscillation grows exponentially. Because of the different values of phase advance in the horizontal and vertical planes we should keep the field appropriate for both planes. Therefore it is reasonable to have the phase advance $\sim 75^{\circ}$.

Finally, we shall consider the matching in the transverse planes. There are two possibilities of how to pass from

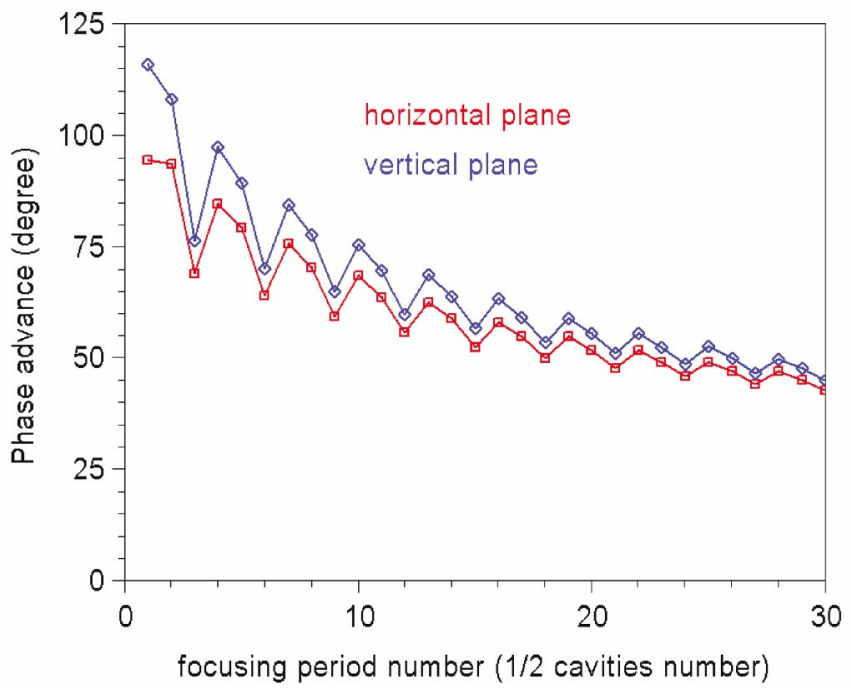

FIG. 13. (Color) Phase advance in both planes.

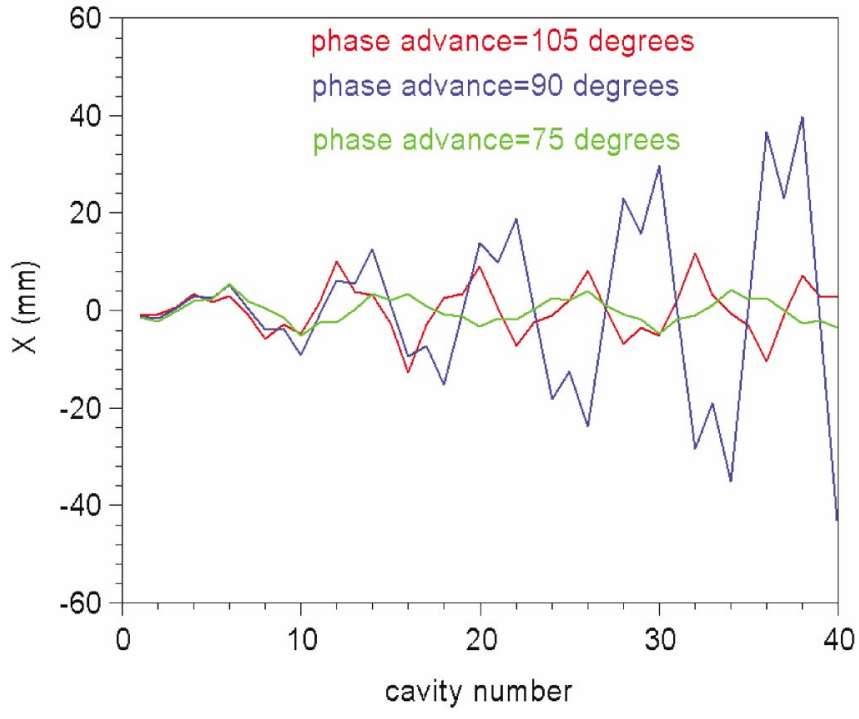

FIG. 14. (Color) Trajectories in the horizontal plane at different phase advance.

cavity to cavity in the linear accelerator with the family grouping. In first version, the cavity transient factor is optimized, and it is attempted to make the structure velocity of the group equal to the quasisynchronous particle in the central cavity or between two central cavities (see Fig. 15). Because of this fact the maximum transient time is reached, namely, in the center of the family. However, in this version the rf phase of the quasisynchronous particle in neighboring cavities takes a different meaning, which is in contradiction to the transverse matching (see Fig. 16). In the second version, the transition between neighboring cavities takes place at equal rf phase at the exit of the previous cavity and the entrance to the

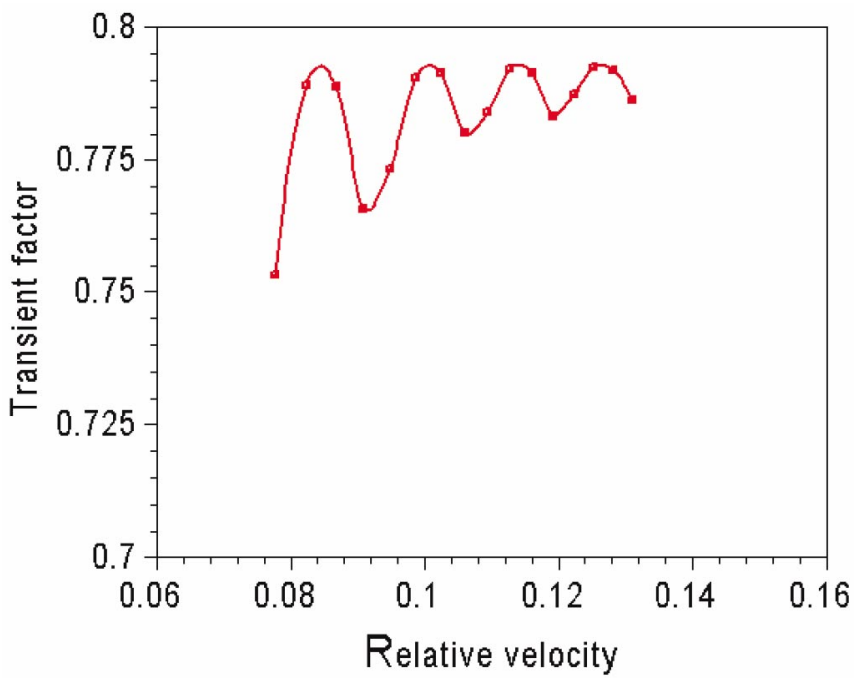

FIG. 15. (Color) Transient factor vs relative velocity for the first version of cavity-cavity matching. 


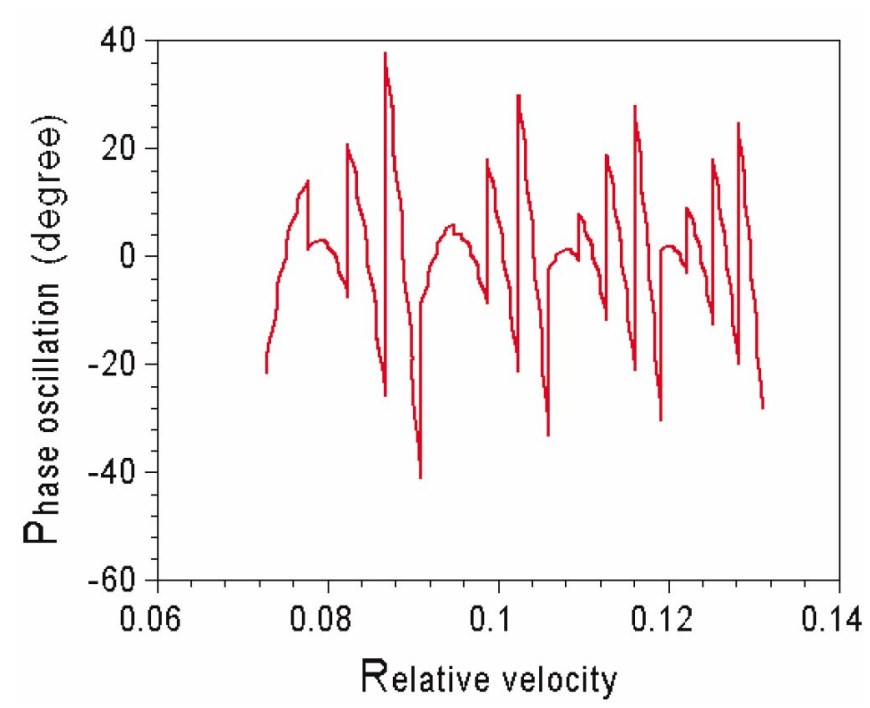

FIG. 16. (Color) Phase oscillation of the quasisynchronous particle vs relative velocity for the first version of the cavity-cavity matching.

next cavity (see Figs. 17 and 18). The 3D tracking shows the latter is preferable also with respect to longitudinal motion.

\section{NUMERICAL SIMULATION}

\section{A. Low-intensity beam}

The beam dynamic is simulated using the 3D PIC code [8] in the 3D electrical and magnetic fields exported from CST Microwave Studio. The initial Gaussian distribution is generated in the 3D ellipsoidal bunch with 100000 particles. In the longitudinal plane, the total emittance is taken to be $0.826 \mathrm{MeV} \operatorname{deg}\left(6.51 \times 10^{-12} \mathrm{MeV} \mathrm{sec}\right)$ with the

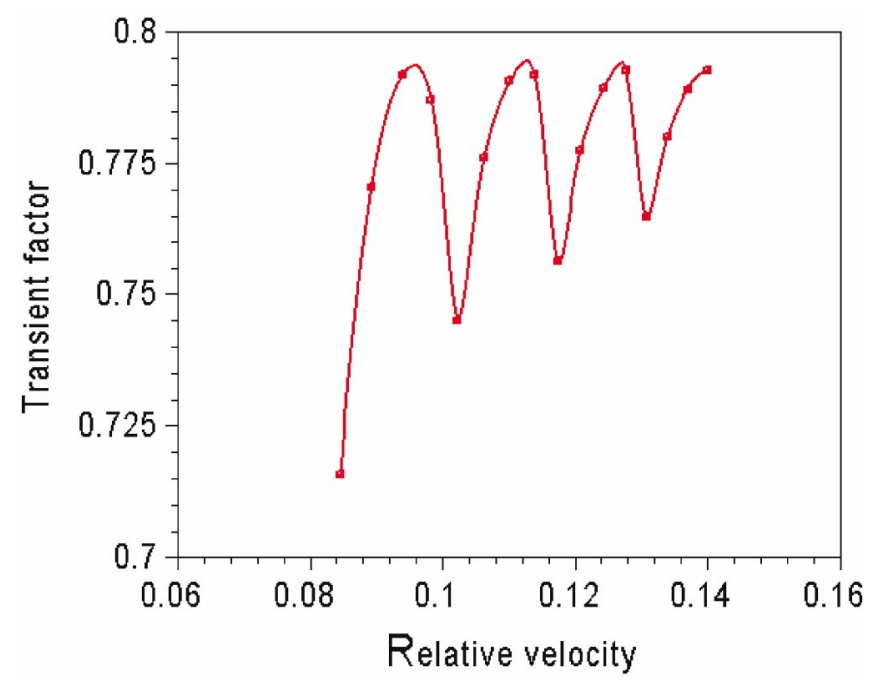

FIG. 17. (Color) Transient factor vs relative velocity for the second version of cavity-cavity matching.

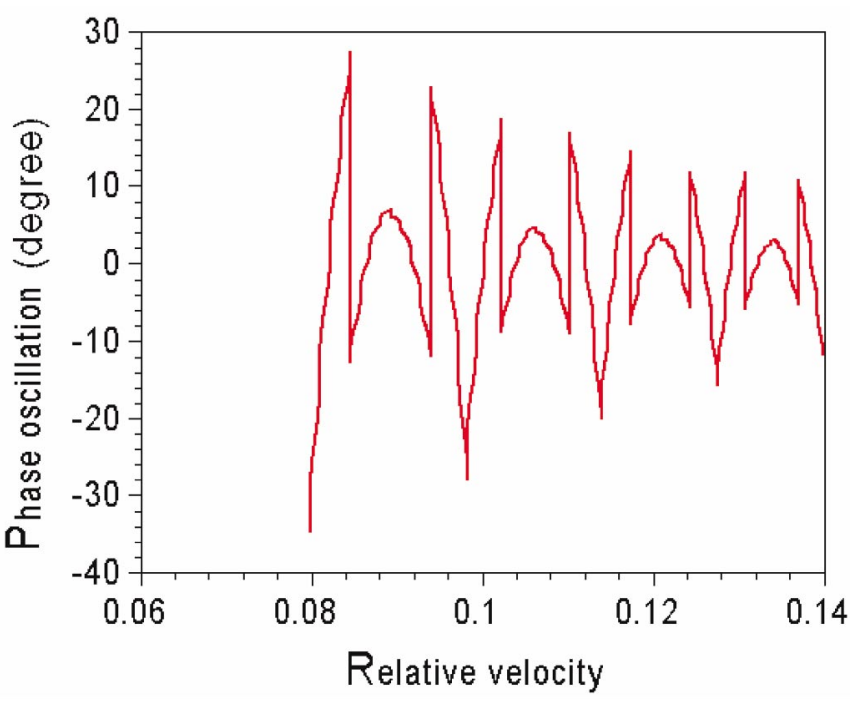

FIG. 18. (Color) Phase oscillation of the quasisynchronous particle vs relative velocity for the second version of cavity-cavity matching.

phase spread $\pm 15^{\circ}(352.2 \mathrm{MHz})$ and the energy spread $\pm 0.05 \mathrm{MeV}$ at energy $3 \mathrm{MeV}$, and it is truncated at 1 sigma in order to make it similar to the more realistic parabolic distribution. In the transverse plane, the normalized rms emittance is assumed to be equal to $0.33 \pi \mathrm{mm} \mathrm{mrad}$ with the total emittance of $1.32 \pi \mathrm{mm}$ mrad expecting collimation of the beam at $2 \sigma$.

The accelerating channel is based on the slot-finger structure with the FOODDOOF lattice [see Fig. 11(b)]. Carrying the rf electrodes inside the cavity, the accelerating gradient is restricted by the peak of the electric field on the rf electrodes. Therefore the accelerating gradient becomes dependent on the electrode configuration. At the first stage of acceleration in the low-energy part $\sim(3-10) \mathrm{MeV}$ the transverse phase advance is kept constant in order to provide stability of the high-intensity beam in both planes. Hence, the distance and the potential between the rf electrodes have to be kept constant as well. As long as the potential is limited by the peak electric field on the electrodes, the energy gain per meter decreases with increasing cavity length proportional to $\sim 1 / \beta$. Taking into account the wave number in Eq. (11), the defocusing rf field already decreases proportional to $\sim 1 / \beta^{2}$. Because of this fact and the proportionality of the structure velocity to the quasisynchronous particle, the longitudinal frequency [Eq. (48)] decreases proportional to $\sim 1 / \beta$. At the second stage of acceleration, when the longitudinal phase advance goes away from the first-order longitudinal-transverse resonance, the distance between the rf electrodes increases proportional to $\sim \beta$. Thus, the potential on the electrodes can be increased to keep the energy gain constant. As a result, the transverse phase advance decreases proportional to $\sim 1 / \beta$, and the maximal aperture radius increases as $\sim \beta$. Following this strategy, the accelerating channel was 


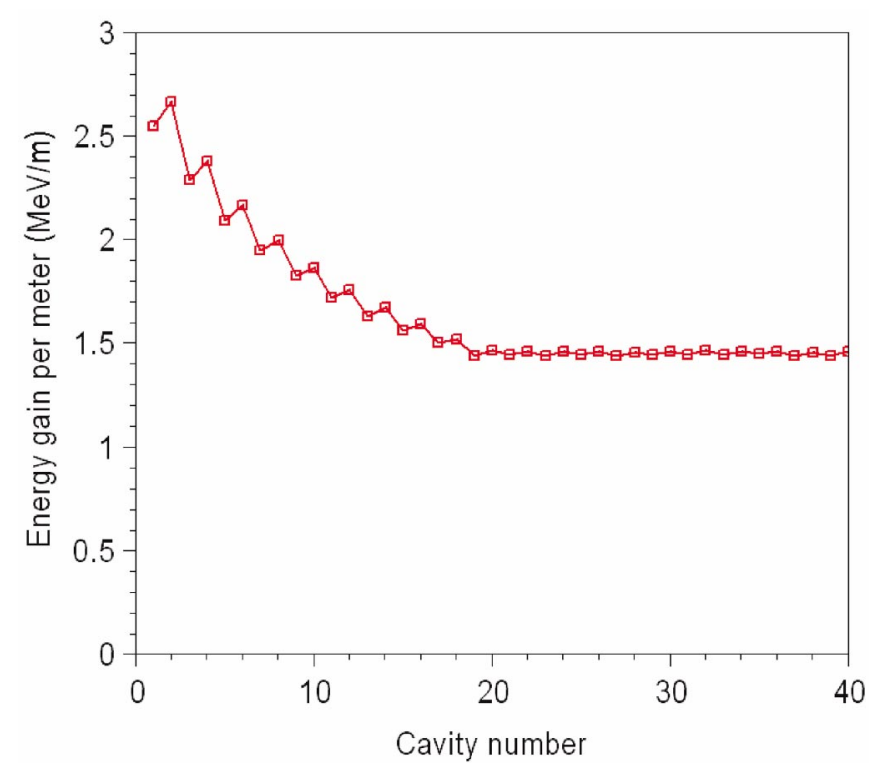

FIG. 19. (Color) Energy gain vs cavity number.

simulated from $3 \mathrm{MeV}$ up to $\sim 20 \mathrm{MeV}$. The energy gain in this geometry is shown in Fig. 19.

As the first step in the investigation, the beam dynamic simulations were performed without the space charge forces. The first option with 6 cavities in one family was tested. Because of the large oscillation and the "familystructure resonance," this option was not accepted.
Therefore the maximum number of cavities in a family is four.

Since the focusing depends on the entrance phase, acceptance is expected to be dependent on the rf phase. Figures 20 and 21 show the acceptance of the accelerating-focusing channel for different rf phases in both planes for the option with 4 cavities in a family.

The common overlapping area shows the total acceptance. Because of the short bunch of $\pm 15^{\circ}$ the influence of the acceptance rf phase dependence is small. The normalized total acceptance for this channel is about $4 \pi \mathrm{mm} \mathrm{mrad}$ (or $50 \pi \mathrm{mm} \mathrm{mrad}$ at initial energy $3 \mathrm{MeV}$, $\beta=0.08)$ in both transverse planes.

By tracking we determined the emittance growth (Fig. 22), the rms envelope (Fig. 23) and the final phase portrait (Fig. 24).

The normalized rms emittance does not grow, but due to the periodicity violation in the focusing channel with the family structure the rms envelope has the essential oscillation even after matching at the linac entrance.

Figure 25 shows the separatrix together with the initial bunch in the relative coordinate $\varphi-\varphi_{q s}$, and Fig. 26 demonstrates the bunch propagation in the absolute coordinate $\varphi$ through all the cavities.

From Fig. 26 we can see the sliding effect in the longitudinal plane and even at $\pm 30^{\circ}$ oscillation does not play a major role, so that the bunch holds its shape during accelerating [4].

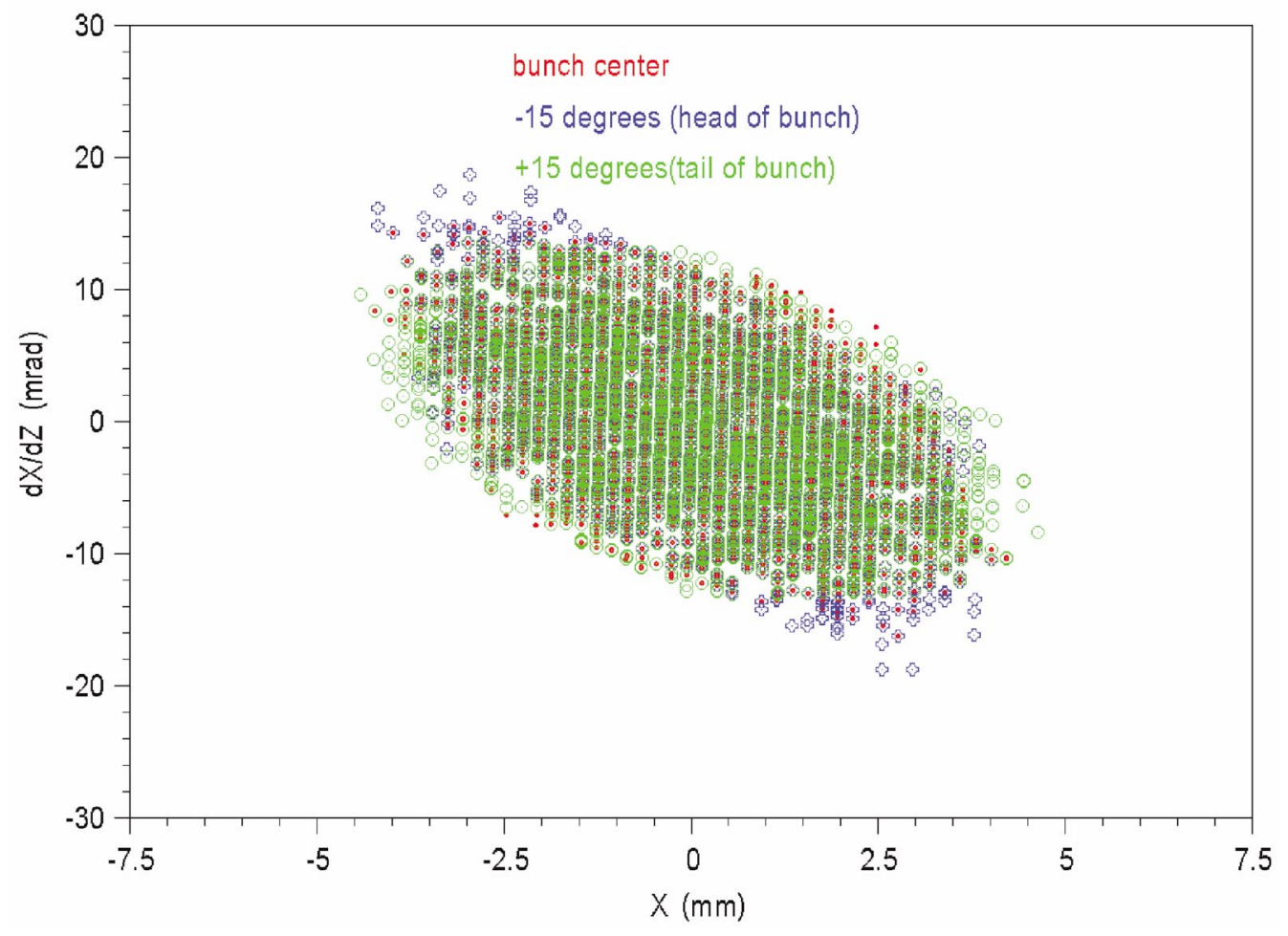

FIG. 20. (Color) Horizontal acceptance vs cross-section position. 


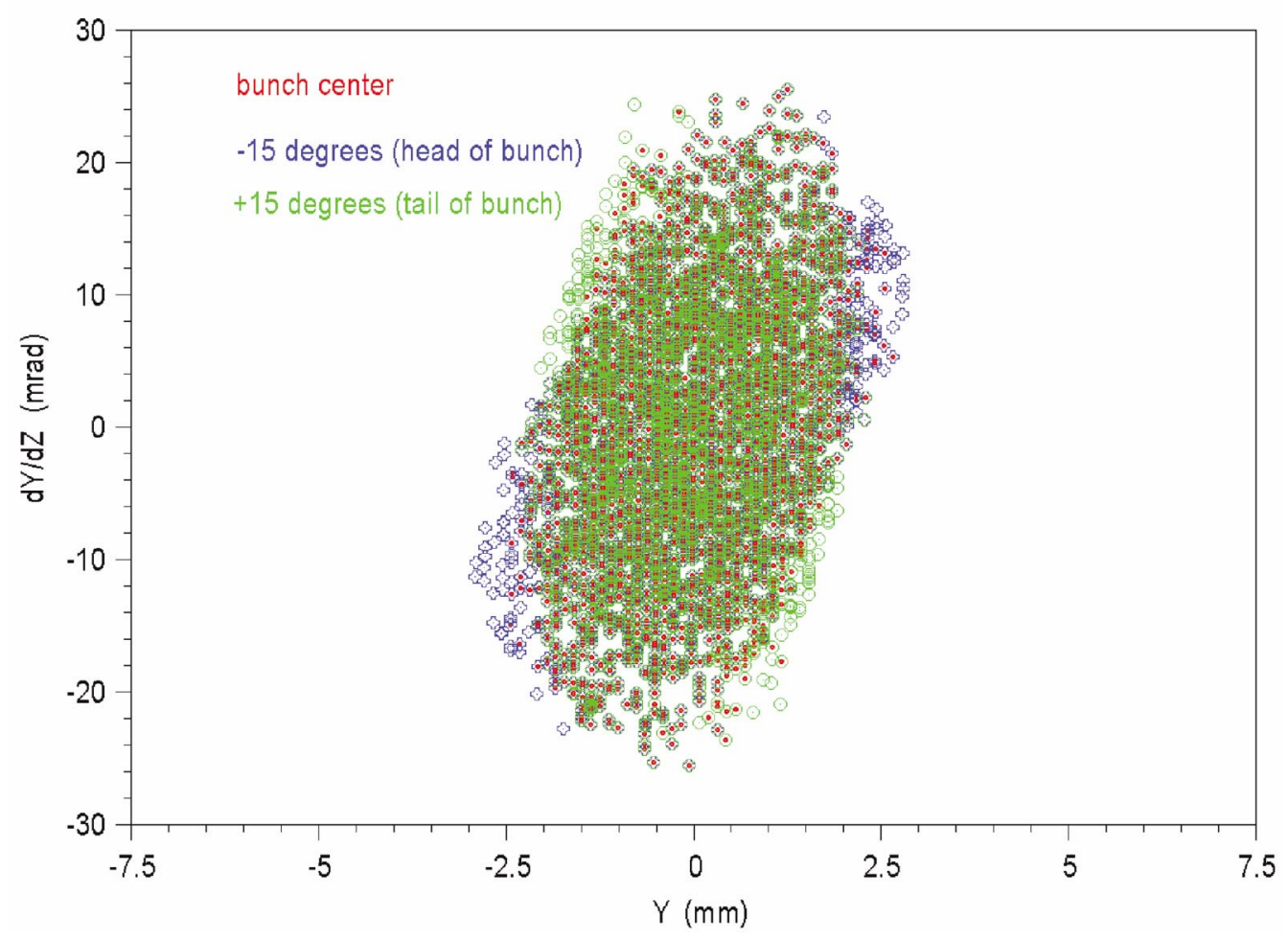

FIG. 21. (Color) Vertical acceptance vs cross-section position.

\section{B. High-intensity beam}

Because of periodicity violation and mismatching, the SC linear accelerator with the "family structure" is more sensitive to the space charge effect in the transverse planes. First of all, we analyzed the option with 4 cavities in a family and found no losses up to $30 \mathrm{~mA}$.

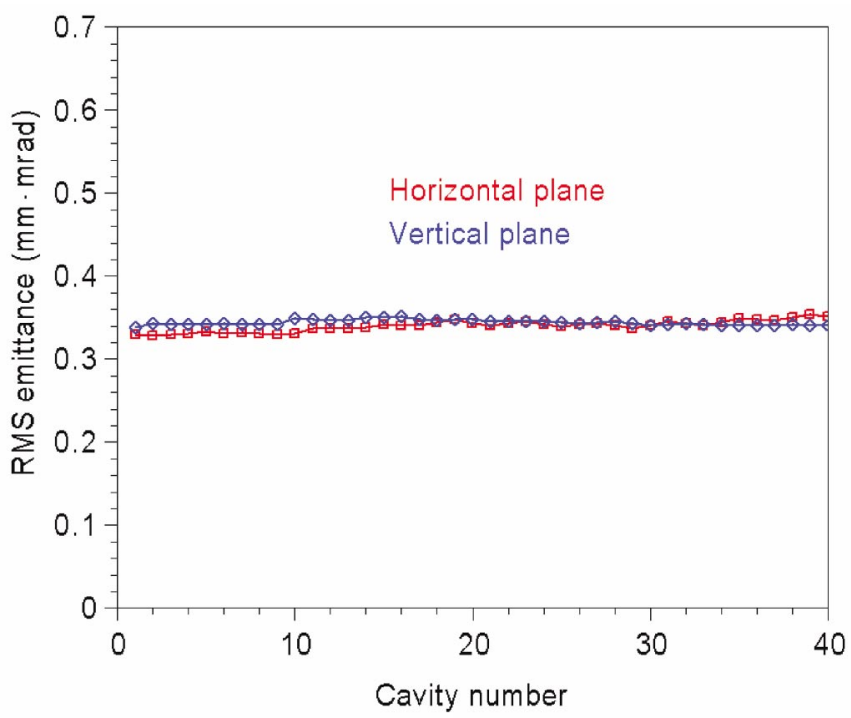

FIG. 22. (Color) rms emittance versus cavity number for zero current.
For the higher intensity, the rms emittance grows due to the envelope beating and the mismatching, and we then observe losses. Therefore, at least in the initial part, the structure with 2 cavities in a family has to be chosen. It is reasonable to take the same average phase of the quasisynchronous particle $\bar{\varphi}_{q s}=-20^{\circ}$. As in the previous case, the structure phase velocity of each family is chosen to mini-

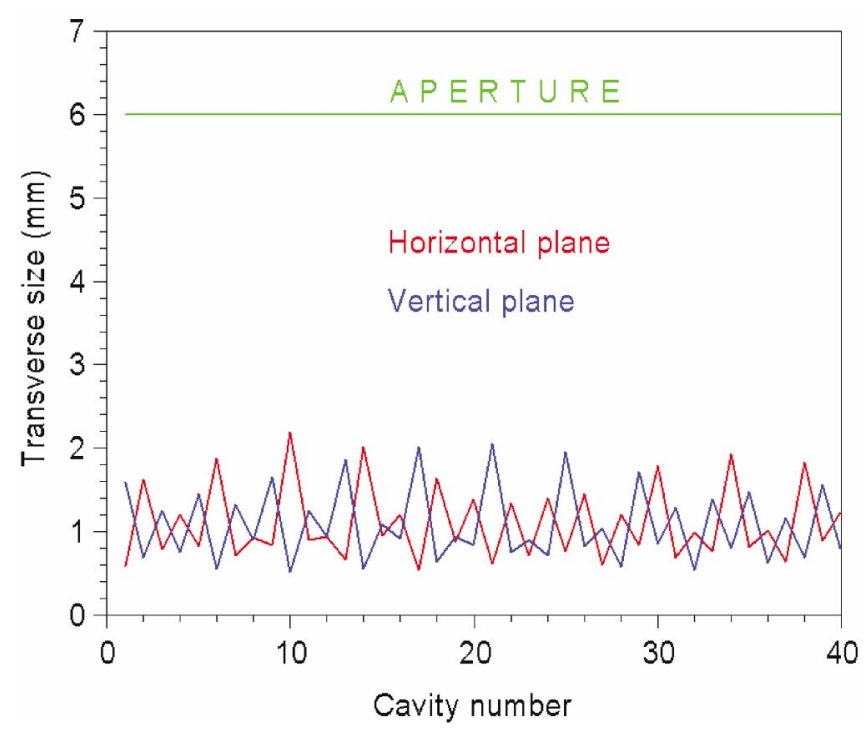

FIG. 23. (Color) rms transverse sizes vs cavity number. 


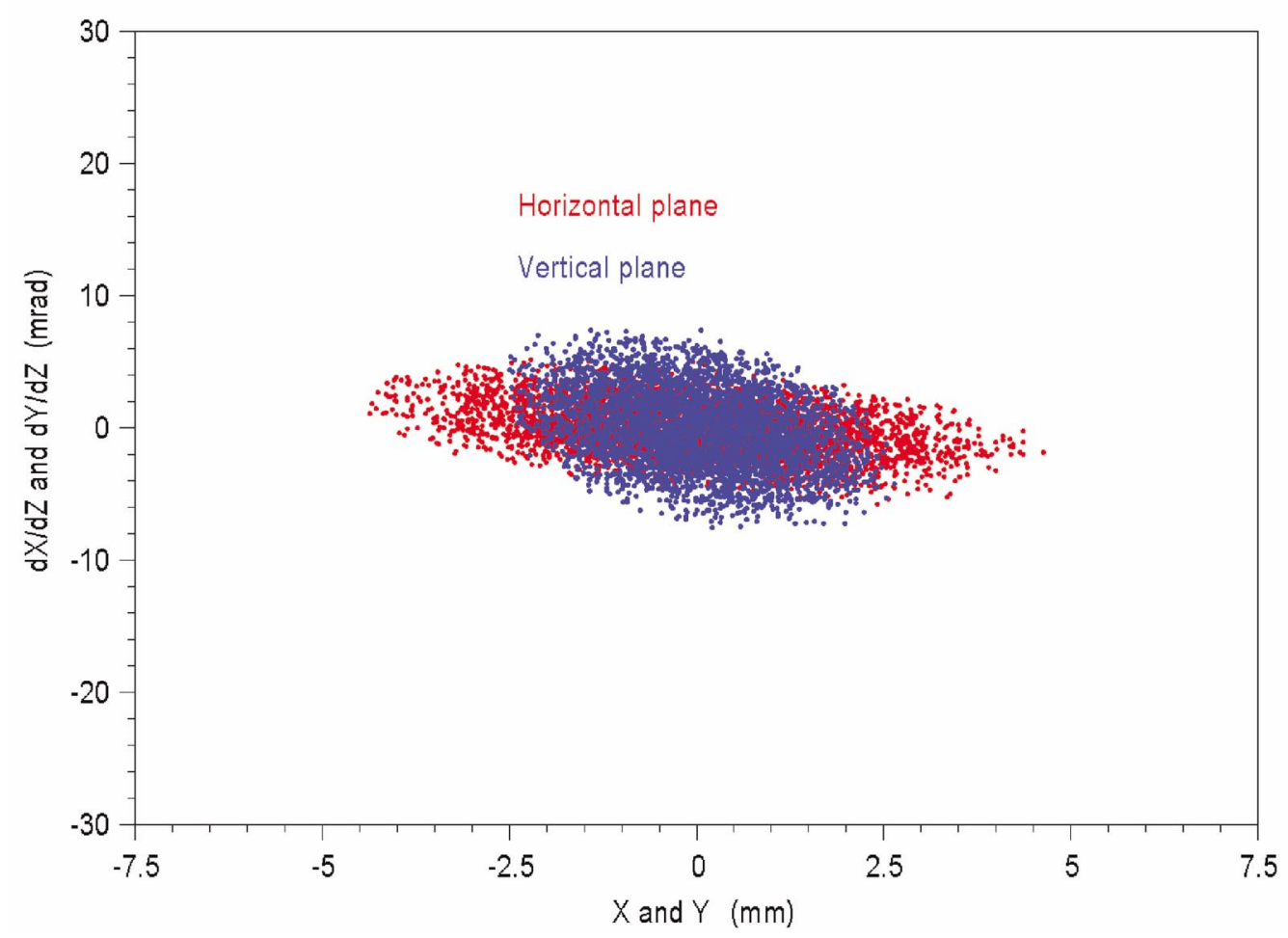

FIG. 24. (Color) Final phase portrait at the linac exit.

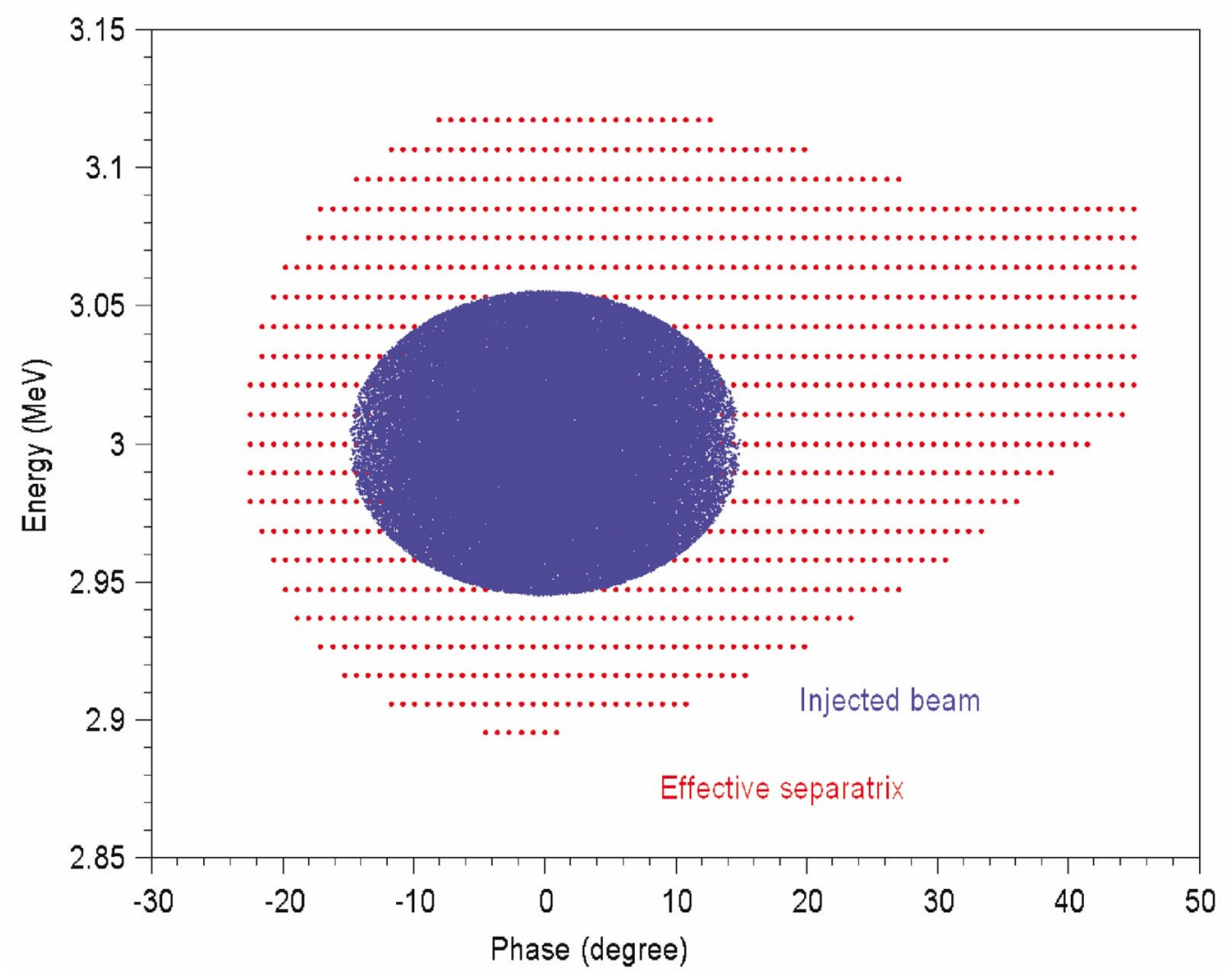

FIG. 25. (Color) Separatrix and initial bunch. 


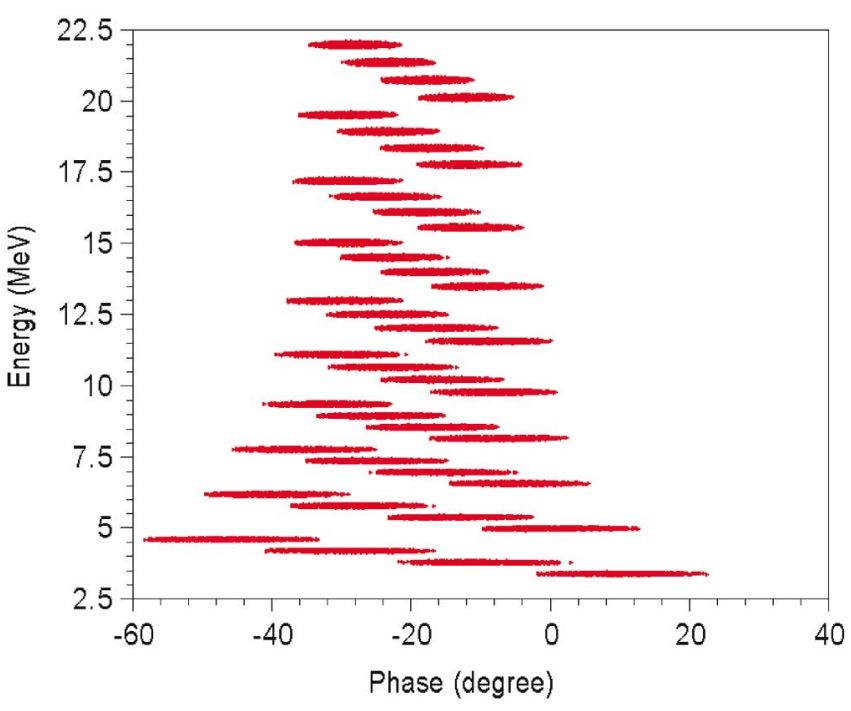

FIG. 26. (Color) Bunch propagation through the linac.

mize sliding of particles relative to the rf wave due to nonsynchronism (Fig. 27).

Using the code [8], we investigated the beam dynamics with the space charge. Figure 28 shows the rms sizes of the beam at $40 \mathrm{~mA}$ versus the cavity number. Despite the matched envelope behavior the normalized transverse emittance grows by almost 50\% (see Fig. 29).

Because of small quasisynchronous phase the space charge tune shift in the longitudinal plane leads to a strong decrease of longitudinal phase advance. Nevertheless, the longitudinal motion remains stable and the bunch conserves the elliptical form (Figs. 30-32). The maximal

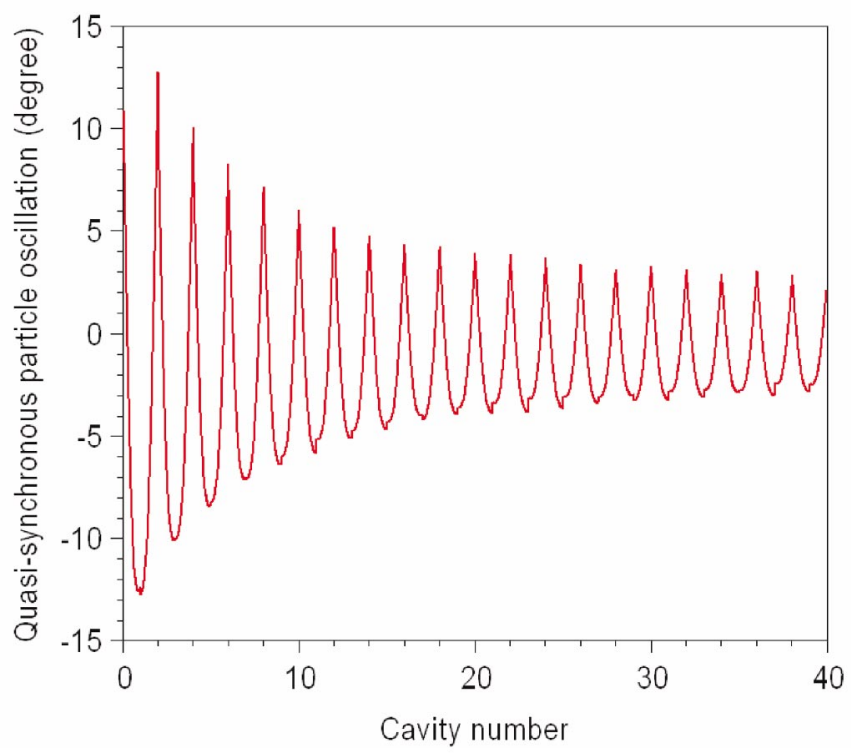

FIG. 27. (Color) Phase of the quasisynchronous particle in the linac with 2 cavities in each family.

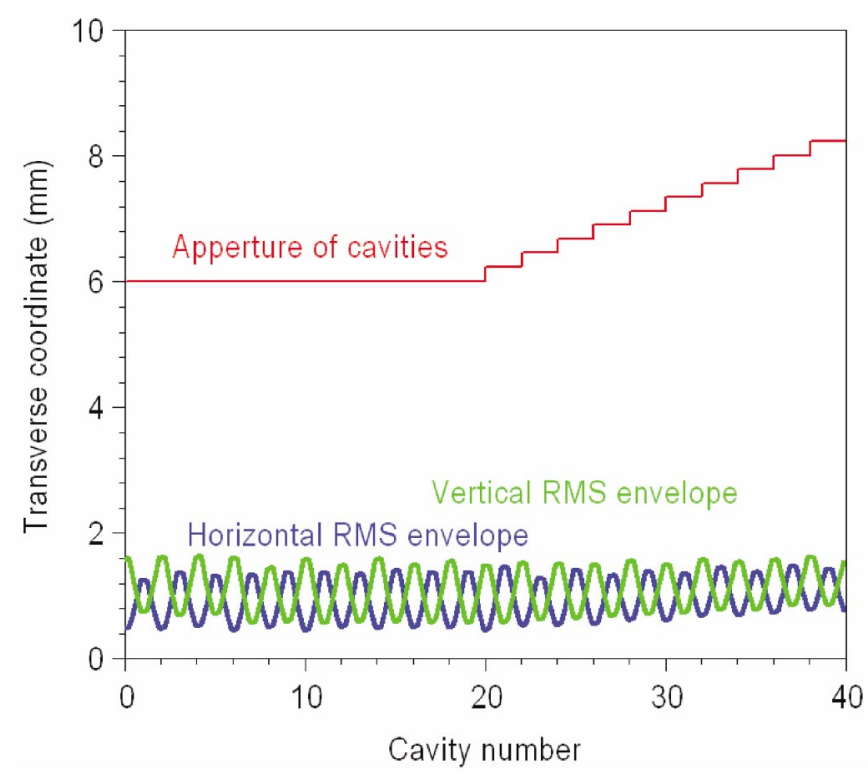

FIG. 28. (Color) rms transverse sizes of the beam at $40 \mathrm{~mA}$ vs cavity number.

deviation of particles during acceleration coincides with the minimal aperture of cavities in the first part of acceleration. However, at the end of acceleration the aperture is significantly larger than the maximal deviation of particles.

\section{CONCLUSION}

We have developed a new accelerating structure, which can be applied for the low-energy proton or the ion linear accelerator. The structure called the slot structure is based on the $\mathrm{H}$ resonator with fundamental mode TE211 loaded

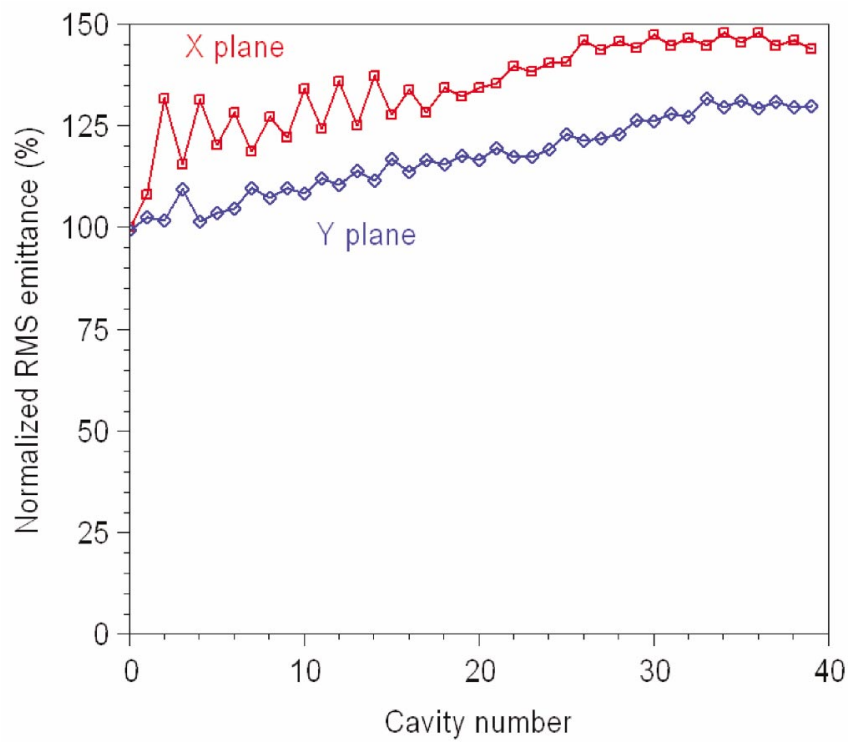

FIG. 29. (Color) rms emittance growth in percentages at $40 \mathrm{~mA}$. 


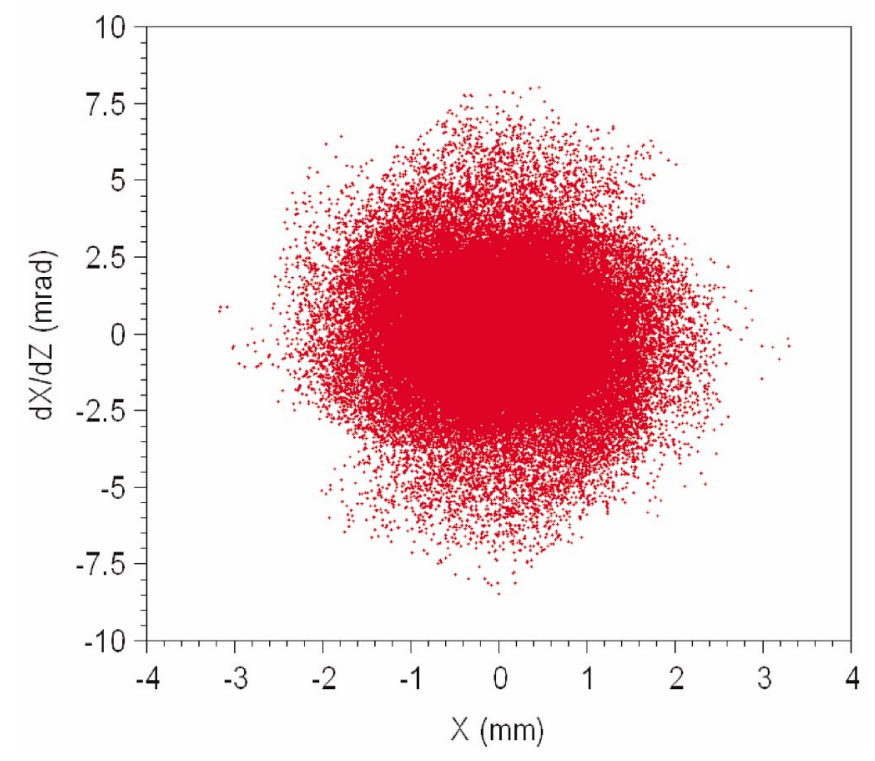

FIG. 30. (Color) Phase portrait in the horizontal plane at the linear accelerator exit.

by stems. The quadrupole component is created in accelerating gaps by two stems alternatively situated in the horizontal and vertical planes from gap to gap in the $\mathrm{H}$ resonator. The TE2 11 is excited, thus creating the $\pi$ mode in the longitudinal plane with period $\beta \lambda / 2$ and the alternative $\mathrm{rf}$ quadrupole field with period $\beta \lambda$.

To increase the focusing and provide stable motion without the quadrupoles we installed two pairs of fingers in the first and the last gaps of the resonator with four accelerating periods. This cavity is called the slot-finger

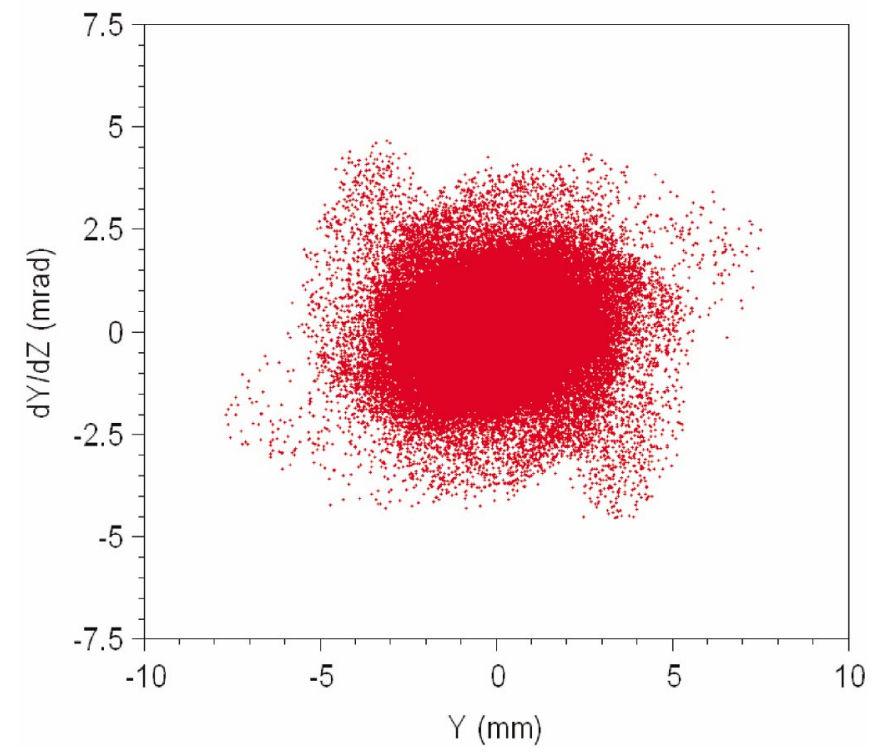

FIG. 31. (Color) Phase portrait in the vertical plane at the linear accelerator exit.

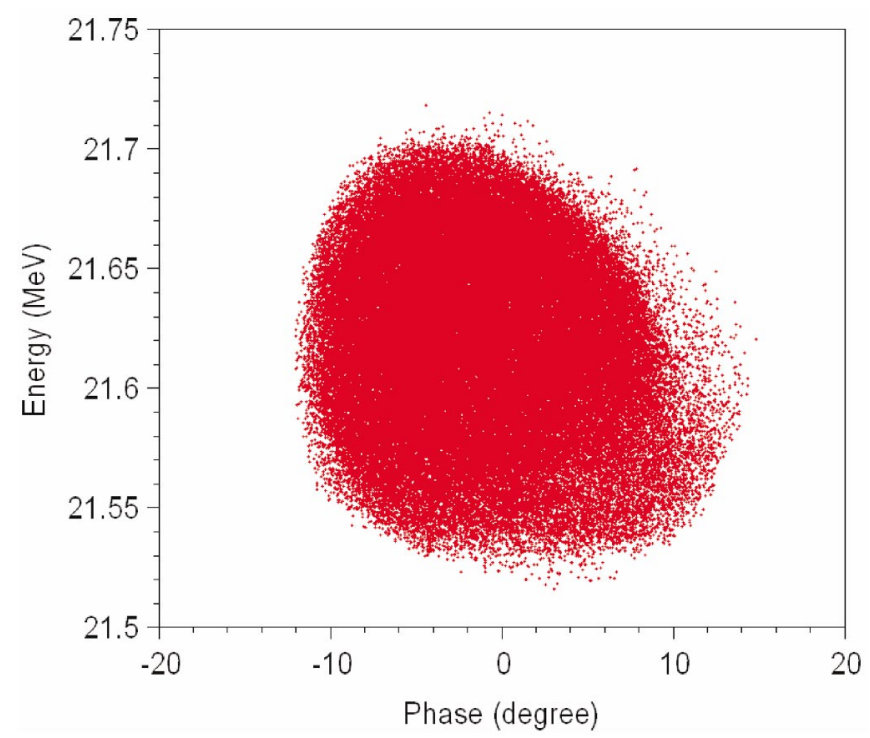

FIG. 32. (Color) The phase portrait in the longitudinal plane at the linear accelerator exit.

resonator. Every other resonator is rotated around the axis to create a FOODDOOF focusing system, giving more stability in the transverse plane.

We investigated the electrodynamics and the beam dynamics in the slot and slot-finger structures. We developed the theory, which allows the machine parameters to be optimized. The numerical investigation was performed by using the $3 \mathrm{D}$ code with space charge.

We can recommend using the slot-finger structure since $3 \mathrm{MeV}$ up to (30-50) $\mathrm{MeV}$ or in the range of the relative velocity $\beta=(0.08-0.25)$. Then the slot structure with the external quadrupoles can be used successfully.

\section{ACKNOWLEDGMENTS}

We acknowledge the support of the European Community-Research Infrastructure Activity under the FP6 "Structuring the European Research Area" program (CARE, Contract No. RII3-CT-2003-506395). The authors would like to thank R. Maier and R. Toelle for support of this work and V. Teplyakov for scientific discussion.

[1] V. A. Teplyakov, Prib. Tekh. Eksp. 6, 24 (1964).

[2] Yu. Budanov et al., in Proceedings of the Linear Accelerator Conference, Lübeck, Germany, 2004, pp. 285-287, http://bel.gsi.de/linac2004/PAPERS/ TUP01.PDF.

[3] G. Bisoffi et al., in Proceedings of the 8th European Particle Accelerator Conference, Paris, 2002 (EPS-IGA and CERN, Geneva, 2002), pp. 266-268, http://accelconf.web.cern.ch/AccelConf/e02/papers/ WEBLA001.PDF 
[4] Yu. Senichev, A. Bogdanov, and R. Maier, Phys. Rev. ST Accel. Beams 6, 124001 (2003).

[5] N. Bogolyubov and Yu. Mitropo'lskij, Asymptotic Methods in the Theory of Non-linear Oscillations (Hindustan Publ., Delhi, 1961).

[6] K.W. Shepard et al., in Proceedings of the Particle Accelerator Conference, New York, 1999 (IEEE, New York, 1999), pp. 955-957, http://accelconf.web.cern.ch/ AccelConf/p99/papers/MOP123.PDF
[7] U. Ratzinger, in Proceedings of the European Particle Accelerator Conference, Vienna, 2000 (EPS, Vienna, 2000), pp. 98-102, http://accelconf.web.cern.ch/ AccelConf/e00/papers/TUZF204.PDF

[8] N. Vasyukhin, R. Maier, and Yu. Senichev, in Proceedings of the European Particle Accelerator Conference, Lucerne, 2004 (EPS-AG, Lucerne, 2004), pp. 2002-2004, http://accelconf.web.cern.ch/AccelConf/e04/ PAPERS/WEPLT067.PDF 